\title{
WestVirginiaUniversity
}

THE RESEARCH REPOSITORY @ WVU

Graduate Theses, Dissertations, and Problem Reports

2005

\section{Models and protocols for evaluation of fingerprint sensors}

\author{
Gaurav Gupta \\ West Virginia University
}

Follow this and additional works at: https://researchrepository.wvu.edu/etd

\section{Recommended Citation}

Gupta, Gaurav, "Models and protocols for evaluation of fingerprint sensors" (2005). Graduate Theses, Dissertations, and Problem Reports. 4153.

https://researchrepository.wvu.edu/etd/4153

This Thesis is protected by copyright and/or related rights. It has been brought to you by the The Research Repository @ WVU with permission from the rights-holder(s). You are free to use this Thesis in any way that is permitted by the copyright and related rights legislation that applies to your use. For other uses you must obtain permission from the rights-holder(s) directly, unless additional rights are indicated by a Creative Commons license in the record and/ or on the work itself. This Thesis has been accepted for inclusion in WVU Graduate Theses, Dissertations, and Problem Reports collection by an authorized administrator of The Research Repository @ WVU. For more information, please contact researchrepository@mail.wvu.edu. 
Models and Protocols for Evaluation of Fingerprint

Sensors

Gaurav Gupta

Thesis Submitted to the

College of Engineering and Mineral Resources

at West Virginia University

in partial fulfillment of the requirements

for the degree of

Master of Science

in

Electrical Engineering

Bojan Cukic Ph.D., Chair

Arun A. Ross Ph.D.

Xin Li Ph.D.

Lane Department of Computer Science and Electrical Engineering

\section{Morgantown, WV}

2005

Keywords: Biometrics, Fingerprint, Testing, Protocol, Modeling, Optical Sensor, Capacitive Sensor 


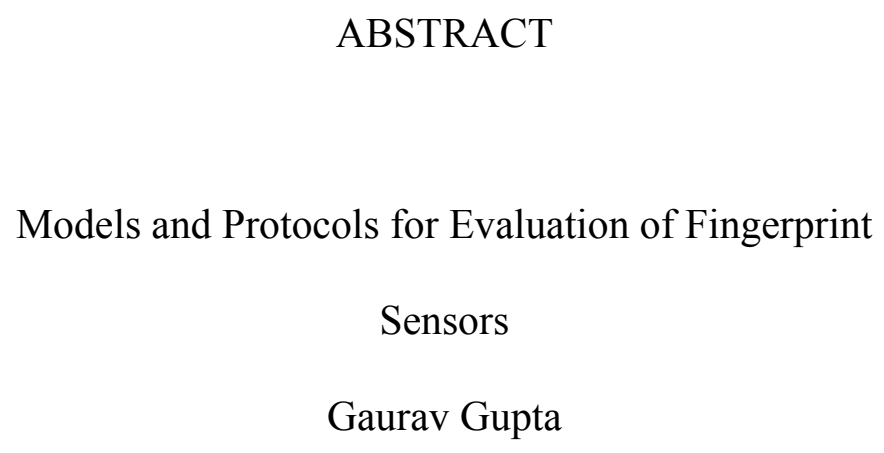

Generic and repeatable testing protocols that can be applied to any biometric system are essential for the development of standards in biometric system testing. The purpose of this thesis is to summarize the current understanding in the biometrics community of the best scientific practices for conducting technical performance testing for purposes of field performance estimation. The thesis identifies various scenarios affecting the performance of a general fingerprint recognition system and compares the performance of an optical and capacitive sensor in these scenarios using the developed protocols. Modeling of these performance characteristics is done in order to predict the performance of the system.

The results of this research suggest that the scenarios considered here do affect the performance of a fingerprint device. However, the affect depends on the type of sensor used. The analysis indicates that optical fingerprint sensors are more robust to sensor cleaning compared to capacitive sensors. On the other hand, temperature is an issue with optical sensors and not with capacitive sensors. 
To my mother, Indu Gupta

To my father, M. K.Gupta

To my mentors, Dr. Bojan Cukic \& Dr. Arun Ross

To my friends

To the World 


\section{Acknowledgments}

I am eternally grateful to my advisor Dr. Bojan Cukic for his constant support and encouragement. Sir you are the basic reason for the metamorphosis that is taking place in my ability to contemplate and research. I thank you for all the intellectually stimulating conversation and extremely helpful discussions we had.

I wish to express my sincere gratitude to Dr. Arun Ross for his help during my Master's program. Sir, without your help and advice I could not imagine writing this thesis.

I thank the member of the committee Dr. Xin Li for his patience, guidance and valuable inputs. I also thank all the faculty members of WVU CSEE department for all their help and guidance. I thank the National Biometric Security Project, Morgantown, Mr. Ramzi Nassar for putting up with me. I thank my friend Travis for all his help. Finally, I want to thank my parents Mr. and Mrs. Gupta, my brother and my sister in law for their support, blessings and encouragement. 


\section{Table of Contents}

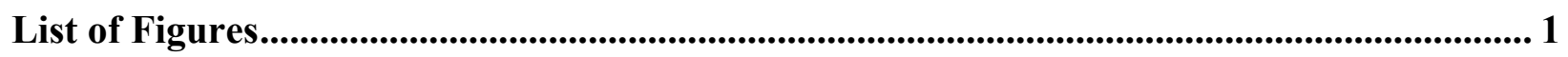

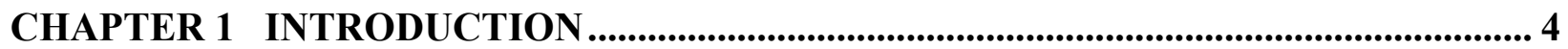

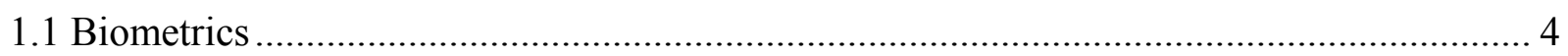

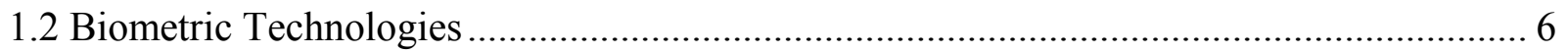

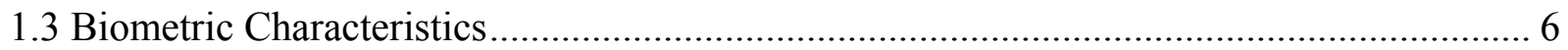

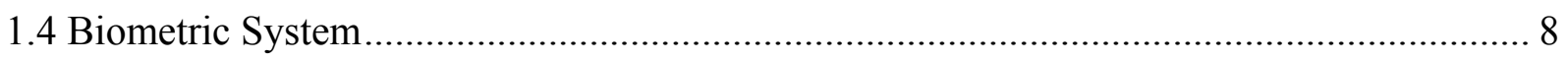

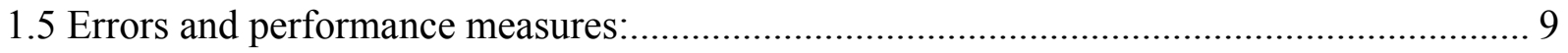

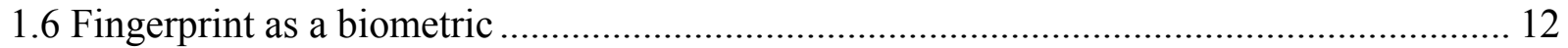

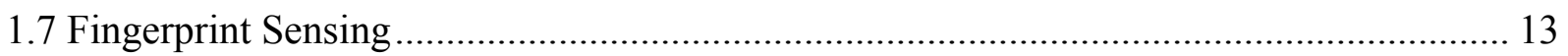

1.8 Biometric system testing and evaluation .................................................................... 15

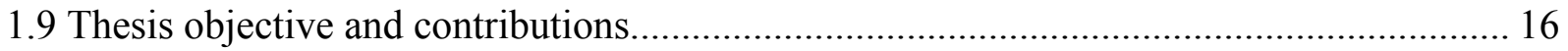

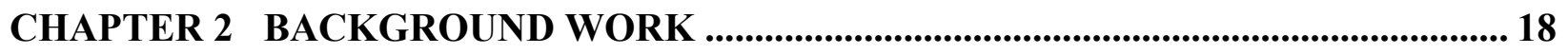

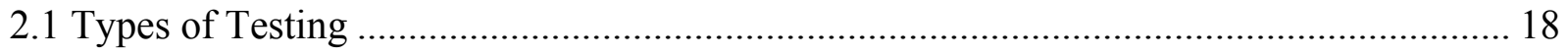

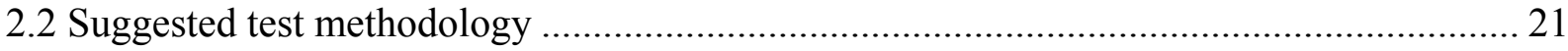

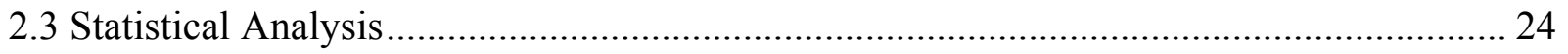

2.4 Evaluation of fingerprint readers in different scenarios .................................................. 27

CHAPTER 3 GENERIC TEST PROTOCOLS .......................................................................... 31

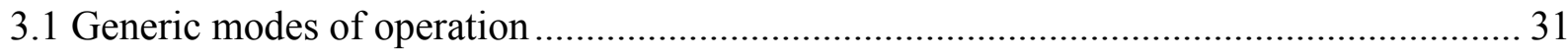

3.2 Variables impacting the generic modes of operation........................................................ 37

CHAPTER 4 EXPERIMENTS..................................................................................................... 40

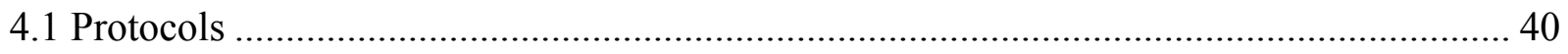

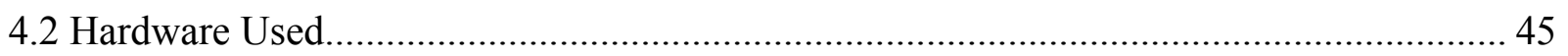

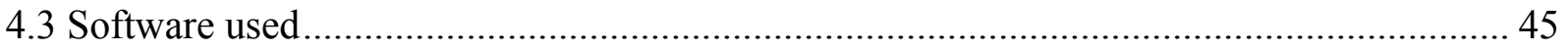

4.4 Testing Protocol for frequency of sensor cleaning .......................................................... 46

4.5 Testing Protocol for indoor outdoor temperature difference ............................................ 49

CHAPTER 5 RESULTS................................................................................................... 52

CHAPTER 6 ANALYSIS AND FUTURE WORK............................................................ 65

6.1 Comparison between optical an capacitive sensor........................................................... 65

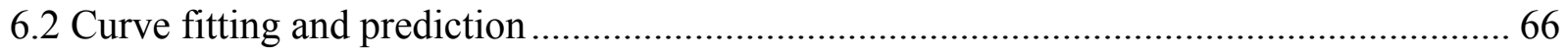

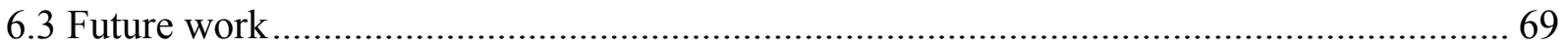




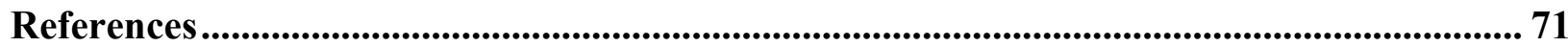

APPENDIX A SecuGen Hamster Optical Fingerprint Sensor .................................................. 76

APPENDIX C Model: THWD-2 Digital Sling Psychrometer....................................................... 77

APPENDIX D Robotic Tripod.................................................................................................... 78 


\section{List of Figures}

Figure $1.1 \quad$ Verification Modes

Figure 1.2 Identification mode

Figure 1.3 Comparison of Biometric Identifiers

Figure 1.4 Generic Biometric Recognition System

Figure 1.5 Equal Error Rate

Figure 1.6 ROC curve

Figure 1.7 Optical Fingerprint Sensor

Figure 1.8 Capacitive Fingerprint sensor

Figure 2.1 Open Set Vs Closed Set Testing

Figure 2.2 Testing Technologies Comparison

Figure 2.3 Affect of Environmental and Human Factors on Optical Fingerprint

Sensor

Figure 2.4 Affect of Environmental and Human Factors on Capacitive Fingerprint

Sensor

Figure 3.1 Acquisition Mode

Figure 3.2 Enrollment Mode

Figure 3.3 Verification Mode

Figure $3.4 \quad$ Identification Mode

Figure 3.5 User Template Update Mode

Figure 4.1 Angles of Rotation

Figure 4.2 Fingerprint Scanning Positions

Figure 4.3 Device Placement (a) Change in $\sigma$ (b) Change in $\Phi$ 
Figure 5.1 Average Genuine Match Score for Number of Sensor touches (Optical Sensor)

Figure 5.2 Average Genuine Match Score for Number of Sensor touches (Capacitive Sensor)

Figure 5.3 Average Number of Minutiae points for Number of Sensor touches (Optical Sensor)

Figure 5.4 Average Number of Minutiae points for Number of Sensor touches (Capacitive Sensor)

Figure 5.5 Average Genuine Match Score for Change in Temperature in Faraheneit (Optical Sensor)

Figure 5.6 Average Genuine Match Score for Change in Temperature in Faraheneit (Capacitive Sensor)

Figure 5.7 Average Number of Minutiae points for Temperature Change (Optical Sensor)

Figure 5.8 Average Number of Minutiae points for Temperature Change (Capacitive Sensor)

Figure 5.9 Average Genuine Match Score for Device Placement (Optical Sensor)

Figure 5.10 Average Genuine Match Score for Device Placement (Capacitive Sensor)

Figure 5.11 Average Number of Minutiae points for Device Placement (Optical Sensor)

Figure 5.12 Average Number of Minutiae points for Device Placement (Capacitive Sensor)

Figure 5.13 Average Genuine Match Score for Biometric Presentation in degrees (Optical Sensor) 
Figure 5.14 Average Genuine Match Score for Biometric Presentation in degrees (Capacitive Sensor)

Figure 5.15 Average Number of Minutiae points for Angle of Rotation (Optical Sensor)

Figure 5.16 Average Number of Minutiae points for Angle of Rotation (Capacitive Sensor)

Figure 6.1 Curve fitting using non linear regression for change in sensor frequency (capacitive sensor)

Figure 6.2 Curve fitting using non linear regression for change in angle of rotation (capacitive sensor)

Figure 6.3 Curve fitting using non linear regression for change in angle of rotation (optical sensor) 


\section{CHAPTER 1 INTRODUCTION}

\subsection{Biometrics}

"Biometrics" is a word that we will hear more and more in the near future. It is derived from Greek words "bios" for life and "metric" for measure. In the realm of computer security it is defined as automatic recognition of a person based on their physiological and behavioral trait. For example fingerprint, face, iris, speech, gait etc. Because of increased emphasis on security due to terrorist attacks, identity thefts, and computer hackers, biometrics has experienced significant growth in the past decade. Although the field is still in its infancy it's inevitable and it will play a crucial role in the security of the future world. The origins of biometrics can be traced to 14th century China for fingerprinting [19] [12]. In the late 1890s, a method of bodily measurement devised by Alphonse Bertillon was used by police authorities throughout the world. This system was flawed in that more than one person could share the same measurement. After that fingerprint a system which was developed by Richard Edward Henry of Scotland Yard became the only biometric identification tool for police.

Verification systems answer the question "Am I who I claim to be". The verification system does 1:1 (one to one) matching. After the user claim an identity he is requested to present their biometric which is then compared to his or her enrolled data. See Fig 1.1 for graphical representation. 


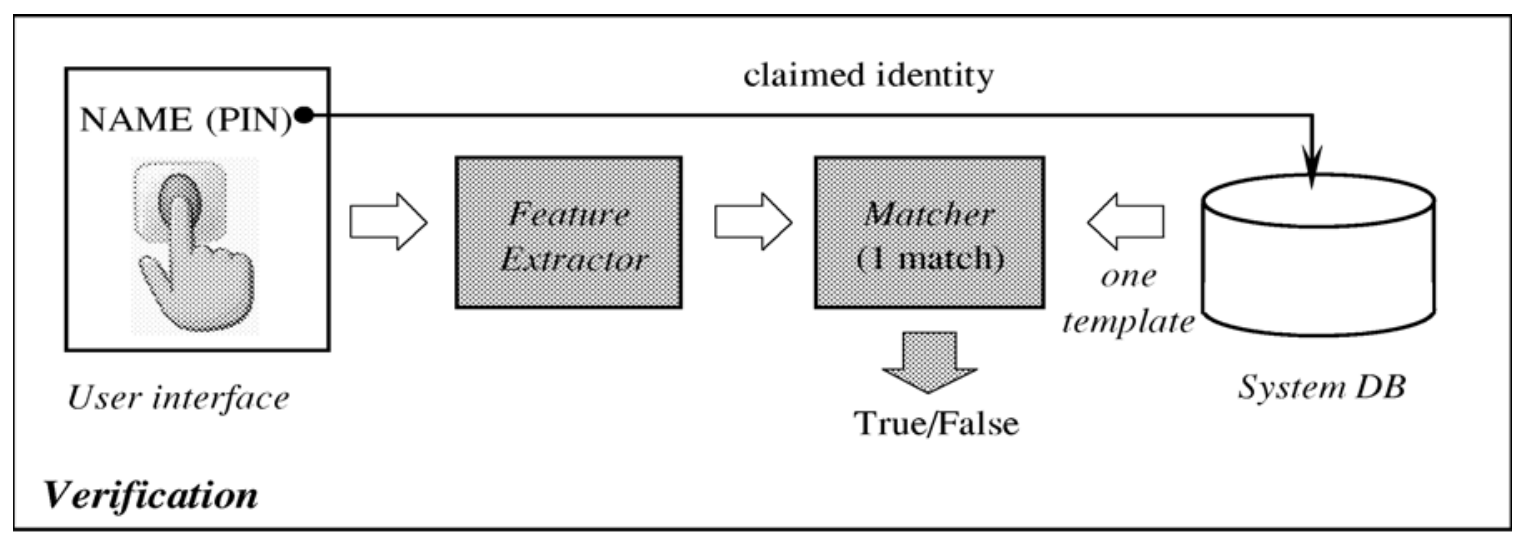

Figure 1.1 Verification Mode [14]

Identification systems answer the question "Who am I". The identification system performs 1: $\mathrm{N}$ (one to many) as shown in Fig 1.2. The user is required to present their biometric and then it is compared to all the users enrolled in the database.

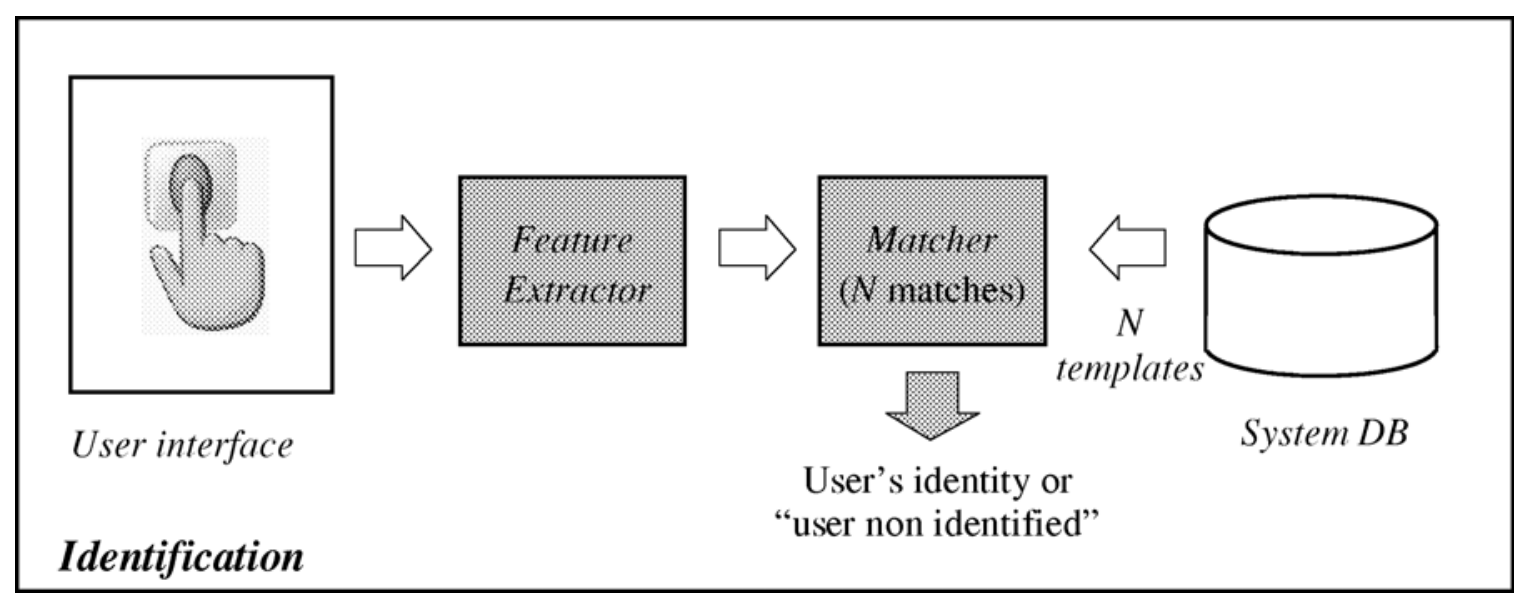

Figure 1.2 Identification Mode [14]

Verification systems are generally faster and simpler than identification systems. Because in verification system comparison is done against only one enrolled data this is computationally less expensive as compared to identification system. 


\subsection{Biometric Technologies}

There are several biometric technologies available in the market using different parts of a human body. Some of these are:

- Fingerprint: Fingerprint recognition systems scan and match the fingerprint pattern for recognition.

- Face: Face recognition systems detect patterns, shapes, and textures in the face.

- Iris: Iris recognition systems scan the surface of the iris to compare patterns.

- Handprint: Recognition of hand or finger systems scans the entire hand or larger parts of the finger and makes a comparison of skin patterns (similar to fingerprint recognition systems). The difference between a fingerprint recognition system and a hand / finger recognition system lies mostly in the size of the scanner and the resolution of the scanning array.

- Retina: Retina recognition systems collect an image of the retina and compare nerve patterns, blood vessels and similar unique features.

- Speech: Voice recognition systems use characteristics of the voice, such as pitch, tone, and frequency.

- Gait: Gait recognition system detects the individuals by characterizing the way someone walks.

\subsection{Biometric Characteristics}

Any human physiological and/or behavioral characteristic can be used as a biometric characteristic [35] as long as it satisfies the following requirements:

- Universality: Every person should possess the biometric characteristic.

- Distinctiveness: No two persons should have the same characteristics. 
- Permanence: The biometric characteristic should not change over time i.e. it should be sufficiently invariant.

- Collectability: The biometric characteristic should be easily collectable.

- Performance: The biometric characteristic should give reasonable performance in terms of recognition accuracy and speed.

- Acceptability: The characteristic should be readily acceptable by the people.

- Circumvention: The characteristic should not be easily spoofed by using fraudulent methods.

Fig 1.3 gives a comparison of biometric identifiers in a tabular form.

\begin{tabular}{|c|c|c|c|c|c|c|c|}
\hline Biometric identifier & 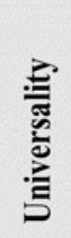 & 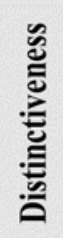 & 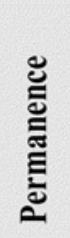 & 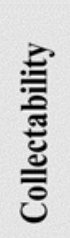 & 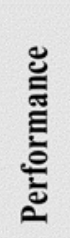 & 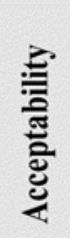 & \\
\hline DNA & $\mathrm{H}$ & $\mathrm{H}$ & $\mathrm{H}$ & $\mathrm{L}$ & $\mathrm{H}$ & $\mathrm{L}$ & $\mathrm{L}$ \\
\hline Ear & M & $M$ & $\mathrm{H}$ & $\mathrm{M}$ & $\mathrm{M}$ & $\mathrm{H}$ & $\mathrm{M}$ \\
\hline Face & $\mathrm{H}$ & $\mathrm{L}$ & $M$ & $\mathrm{H}$ & $\mathrm{L}$ & $\mathrm{H}$ & $\mathrm{H}$ \\
\hline Facial thermogram & $\mathrm{H}$ & $\mathrm{H}$ & $\mathrm{L}$ & $\mathrm{H}$ & $\mathrm{M}$ & $\mathrm{H}$ & $\mathrm{L}$ \\
\hline Fingerprint & $M$ & $\mathrm{H}$ & $\mathrm{H}$ & $M$ & $\mathrm{H}$ & $M$ & $\mathrm{M}$ \\
\hline Gait & $\mathrm{M}$ & $\mathrm{L}$ & $\mathrm{L}$ & $\mathrm{H}$ & $\mathrm{L}$ & $\mathrm{H}$ & $\mathrm{M}$ \\
\hline Hand geometry & $\mathrm{M}$ & $\mathrm{M}$ & $\mathrm{M}$ & $\mathrm{H}$ & $\mathrm{M}$ & $\mathrm{M}$ & $\mathrm{M}$ \\
\hline Hand vein & $\mathrm{M}$ & $M$ & $\mathrm{M}$ & $M$ & $\mathrm{M}$ & $\mathrm{M}$ & $\mathrm{L}$ \\
\hline Iris & $\mathrm{H}$ & $\mathrm{H}$ & $\mathrm{H}$ & $M$ & $\mathrm{H}$ & $\mathrm{L}$ & $\mathrm{L}$ \\
\hline Keystroke & $\mathrm{L}$ & $\mathrm{L}$ & $\mathrm{L}$ & $M$ & $\mathrm{~L}$ & M & $\mathrm{M}$ \\
\hline Odor & $\mathrm{H}$ & $\mathrm{H}$ & $\mathrm{H}$ & $\mathrm{L}$ & $\mathrm{L}$ & $\mathrm{M}$ & $\mathrm{L}$ \\
\hline Palmprint & $\mathrm{M}$ & $\mathrm{H}$ & $\mathrm{H}$ & $M$ & $\mathrm{H}$ & $\mathrm{M}$ & $\mathrm{M}$ \\
\hline Retina & $\mathrm{H}$ & $\mathrm{H}$ & $\mathrm{M}$ & $\mathrm{L}$ & $\mathrm{H}$ & $\mathrm{L}$ & $\mathrm{L}$ \\
\hline Signature & $\mathrm{L}$ & $\mathrm{L}$ & $\mathrm{L}$ & $\mathrm{H}$ & $\mathrm{L}$ & $\mathrm{H}$ & $\mathrm{H}$ \\
\hline Voice & $M$ & $\mathrm{~L}$ & $\mathrm{~L}$ & $M$ & $\mathrm{~L}$ & $\mathrm{H}$ & $\mathrm{H}$ \\
\hline
\end{tabular}

Figure 1.3 Comparison of biometric identifiers [19] 


\subsection{Biometric System}

Fig 1.4 below shows a generic biometric recognition system [20]. It can be divided into five sub systems:

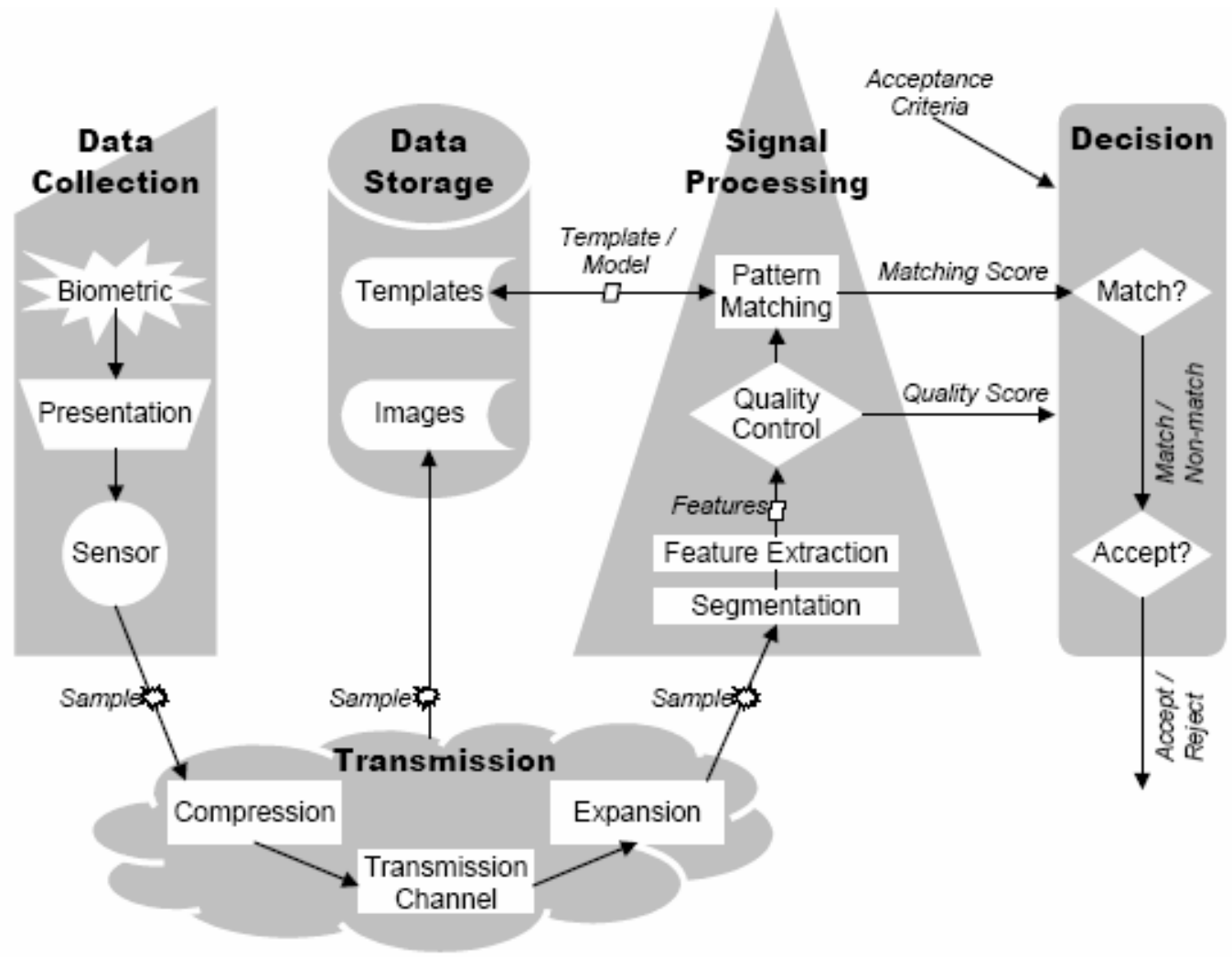

Figure 1.4 Generic Biometric Recognition System [20]

- Data collection: This subsystem acquires the image of the biometric trait of the user. It consists of a sensor that captures the image.

- Transmission: This subsystem receives data from the data collection module, compresses it and then transmits to the signal processing and data storage unit.

- Data Storage: In this subsystem images and templates are stored. 
- Signal Processing: This is the main block of the system. It does all the image processing operation to get the features and also performs pattern matching of extracted features against the stored template and finally generating match scores.

- Decision: This subsystem uses the match score to verify the identity claimed by the user or to identify the user

\subsection{Errors and performance measures:}

- Decision Error Rates are the following[20]:

o False Reject Rate (FRR): The rate at which the system falsely rejects a genuine user compared with total number of trials (Fig. 1.5).

o False Accept Rate (FAR): The rate at which the system falsely accepts an imposter compared with total number of trials (Fig. 1.5).
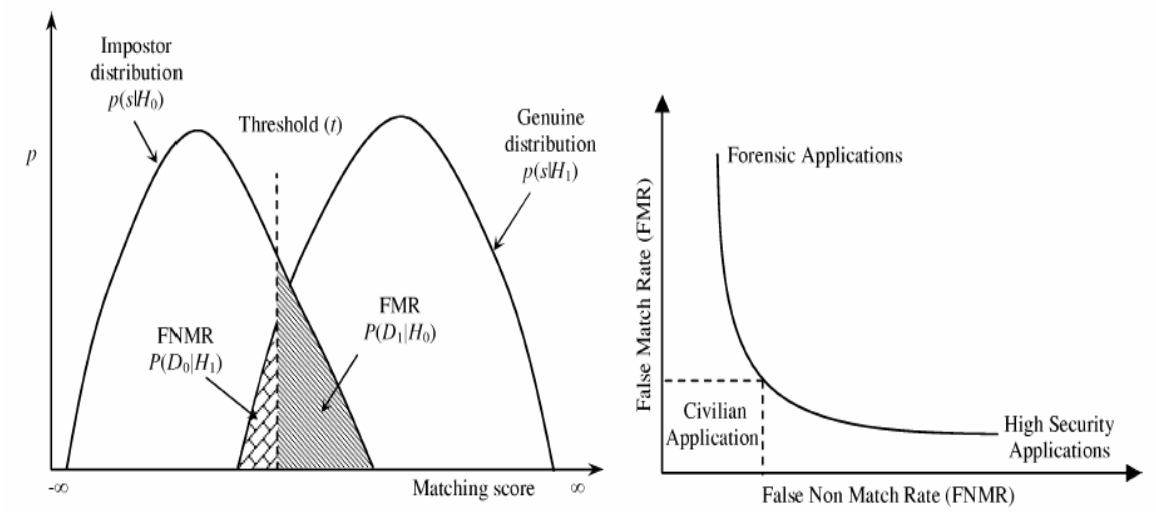

Figure 1.5 Decision Error Rates [13]

o Equal Error Rate (EER): The common value of the FAR and FRR when the FAR equals the FRR. This is the value where both the FAR and FRR are minimized at the same time. A low EER value indicates a high accuracy of the system (Fig. 1.5). 


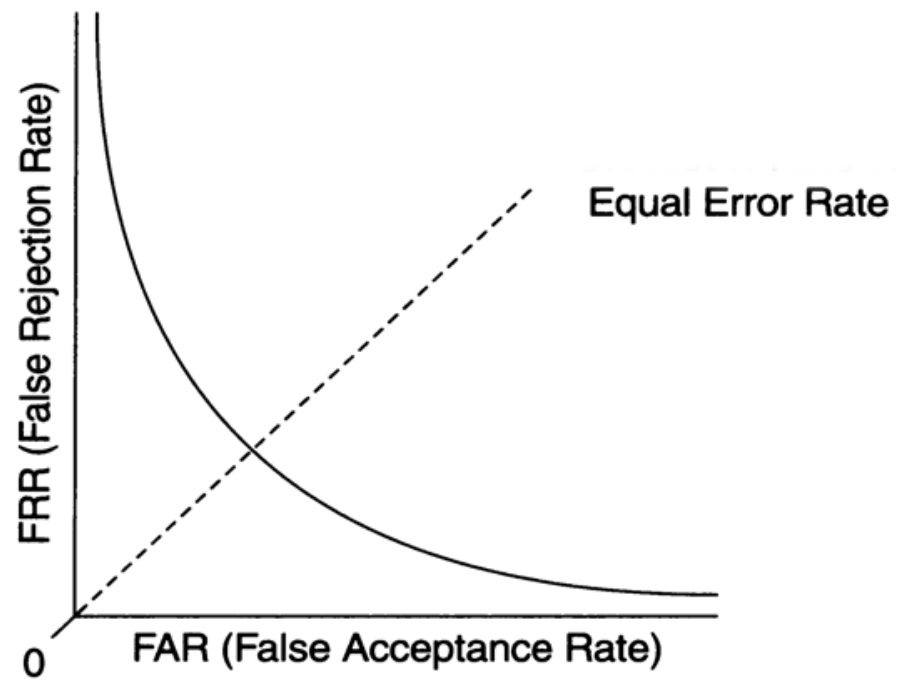

Figure 1.6 Equal Error Rate [19]

o False Non-Match Rate (FNMR): The rate for incorrect negative matches by the matching algorithm for single template comparison attempts. For a biometric system that uses just one attempt to decide acceptance, FNMR is the same as FRR. When multiple attempts are combined in some manner to decide acceptance, FRR is more meaningful at the system level than FNMR (Fig. 1.5).

o False Match Rate (FMR): The rate for incorrect positive matches by the matching algorithm for single template comparison attempts. For a biometric system that uses just one attempt to decide acceptance, FMR is the same as FAR. When multiple attempts are combined in some manner to decide acceptance, FAR is more meaningful at the system level than FMR (Fig. 1.5). 
o Failure to Enroll (FTE): The failure to enroll rate is the proportion of the user population for whom the biometric system is unable to generate reference templates of sufficient quality.

o Failure to Acquire (FTA): The failure to acquire rate is the proportion of attempts for which $a$ biometric system is unable to capture an image of sufficient quality.

- Receiver Operating Characteristics (ROC): A method of showing the performance of the biometric system over a range of decision criteria - usually shown as a graph that relates FAR to FRR as the decision threshold varies.

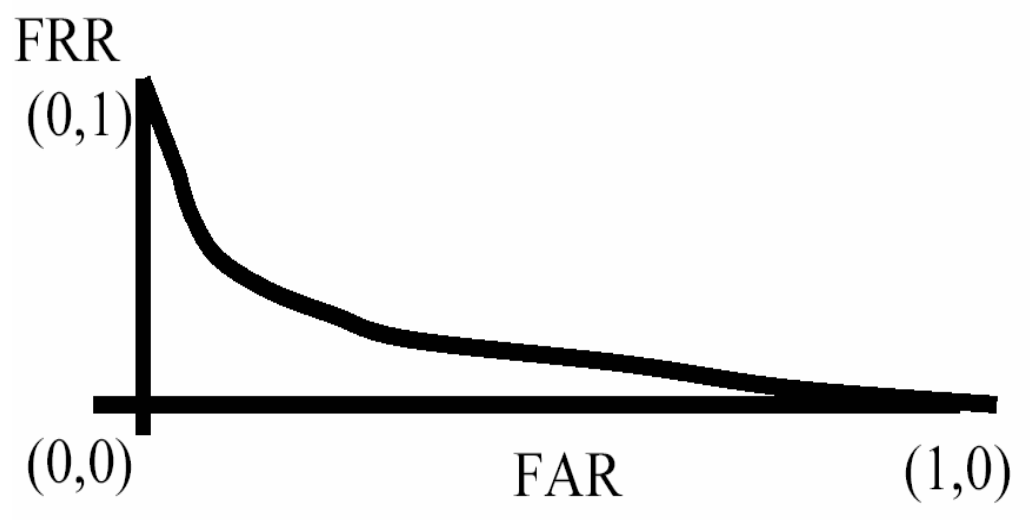

Figure 1.7 ROC Curve [19]

- D-prime measure: A statistical measure of how well a biometric system can discriminate between different individuals. The larger the D Prime value, the better a biometric system is at discriminating between individuals. 
- Detection Error Trade-off (DET): The summarized accuracy of a multimodal biometric system is depicted using the Detection error trade-off (DET) curve, which plots FNMR against FMR directly using logarithmic axes. The DET curve is obtained by ordering the genuine and impostor scores. As the score varies over all possible values, each point on the DET curve represents the false match and false non-match rate using that score as the decision threshold.

\subsection{Fingerprint as a biometric}

Among all the biometric techniques, fingerprint-based identification is the oldest method which has been successfully used in numerous applications. Everyone is claimed to have unique, immutable fingerprints [19] [18]. A fingerprint is made of a series of ridges and furrows on the surface of the finger. The unique features of a fingerprint can be determined by the pattern of ridges and furrows as well as the minutiae points. Minutiae points are local ridge characteristics that occur at either a ridge bifurcation or a ridge ending.

Fingerprints are fully formed at about seven month of fetus development and it does not change through out the life of the individual [19]. This property makes biometric a very attractive identifier.

Although the fingerprint is the most used biometric so far its uniqueness [14] is not an established fact but an empirical observation. Though US supreme court conceded that fingerprints are unique it still lacks an indisputable scientific basis which can establish the fingerprint individuality. 


\subsection{Fingerprint Sensing}

There are number of live-scan devices available in the market that are used to capture the user fingerprint. Some of the most commonly used sensors are optical fulcrated total internal reflection (Optical FTIR), capacitive, thermal, pressure based and ultrasound. In the thesis we used the first two sensors so they are explained in details below:

- Optical FTIR: This is the most widely used sensing technology today [19]. As shown in the Fig 1.8 the finger touches the prism surface and due to that, ridges come into contact to the prism surface while valleys remain at a certain distance. A light source is used to illuminate the prism. The light entering the prism is absorbed by the ridges and reflected at the valleys (due to total internal reflection). The reflected light then is focused by the lens on to an image sensor (CCD or CMOS). Because the reflected light is mostly due to valleys they appear brighter than the ridges.

The advantages with optical sensors include withstanding temperature fluctuations (to some degree), a fairly low cost, resolutions up to $500 \mathrm{dpi}$, better image quality, and the possibility of large sensing areas.

The drawbacks of optical sensors are size and problems with latent fingerprints. Cuts, abrasions, calluses, and other damage, as well as dirt, grease and other contamination, can also be a problem with optical scanners. 


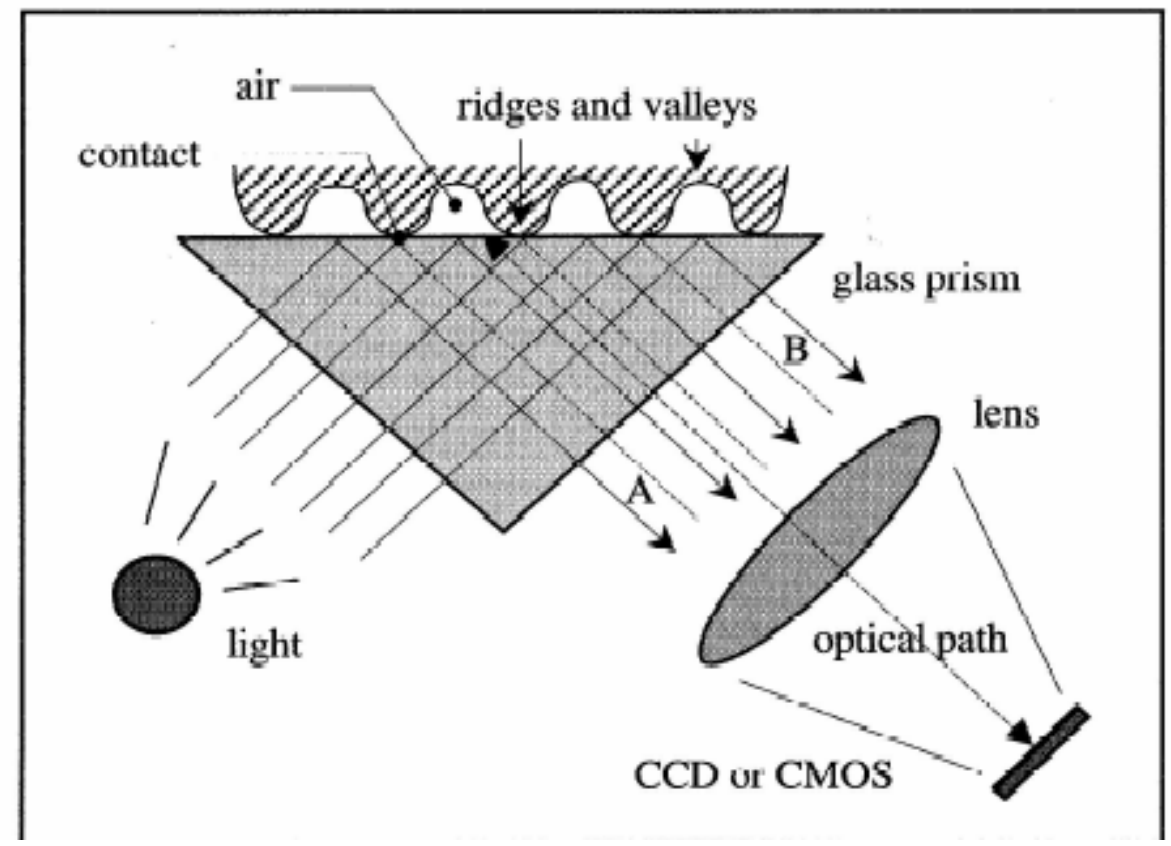

Figure 1.8 Optical Fingerprint Sensor [19]

- Capacitive sensor: These are the most common of the solid state sensors available in the market. As shown in the figure it is a two dimensional array of microcapacitor plates embedded in a chip [19]. When the finger is placed on the plate it acts as the second plate of the micro-capacitor. The electric charges are created between the two capacitor plates and the magnitude of the charge depends upon the distance between the two. Because of different distances of ridges and valleys different capacitive pattern is formed across the plate. 


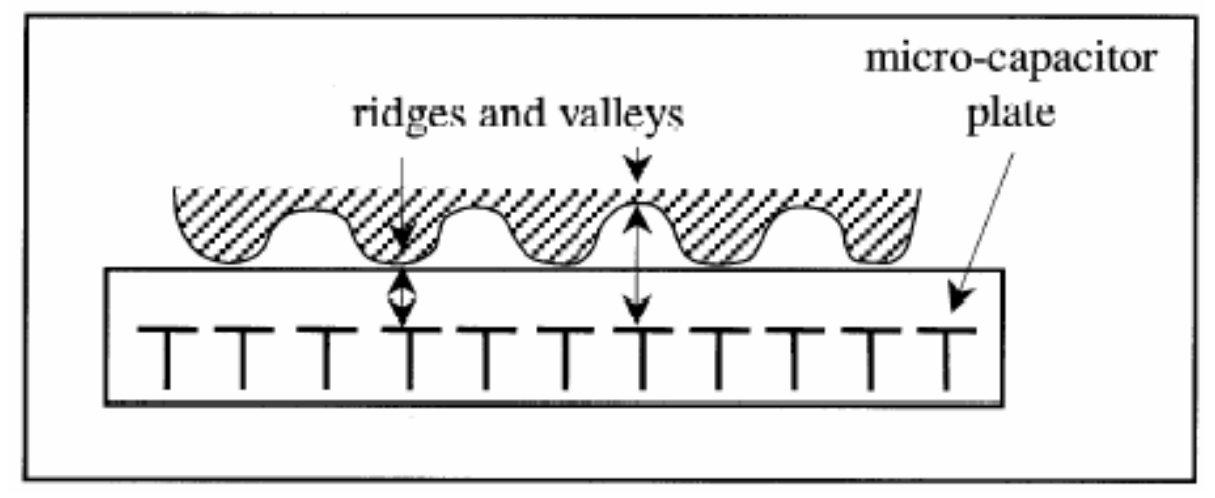

Figure 1.9 Capacitive Fingerprint Sensor [19]

The raw data obtained from these sensors are different [24] due to variations in sensing technology, resolution of the acquired image, area of the sensor, position of the sensor with respect to the user, etc.

\subsection{Biometric system testing and evaluation}

There are three types of evaluation [20] that can be performed on a biometric system

- Technology evaluation: The technology evaluation compares all the algorithms from a single technology. The database that is used to evaluate the algorithms is collected by a "universal sensor". The database used is just right for the algorithms to be tested that is they are collected in an ideal situation. This evaluation process is repeatable as the same database is used every time.

- Scenario evaluation: The scenario evaluation determines the performance of biometric system in an artificially modeled scenario. This scenario is carefully controlled so that the evaluation process can be repeated. As in scenario testing whole biometric system is under evaluation the data acquired by the sensors of each device will be different hence the care should be taken that the same 
environment and population is maintained for all the systems. This is the testing I am interested in, as my thesis is all about scenario testing.

- Operational evaluation: The operational evaluation determines the performance of a whole biometric system in a specific application based environment with a specific target population.

\subsection{Thesis objective and contributions}

The development of a generic set of biometric system test protocols is needed to help in the standardization of performance testing. Testing protocols that can be applied to any biometric system are essential for the development of a standard in biometric system testing. Having broken down a biometric system into its main operating modes and subsequent categories, we consider a set of generic scenarios for testing. This thesis is meant to lay the ground work for further research and testing to determine which scenarios and factors will affect performance results the most. This is done in hope to standardize the testing protocols of biometrics systems and to minimize the amount of testing to be done by eliminating redundant/irrelevant tests. We designed protocols with the following goals in mind:

- Protocols repeatability: To come up with the protocols those are repeatable in every sense because it will allow the ability for testing results to be used as a standard means for comparing biometric systems

- Protocols validity: The aforementioned protocols should be valid so that they can be applied to any device. 
The purpose of this thesis is to summarize the current understanding by the biometrics community of the best scientific practices for conducting technical performance testing toward the end of field performance estimation. Such a document is necessary because even a short review of the technical literature on biometric device testing over the last two decades or more reveals a wide variety of conflicting and contradictory testing protocols. Even single organizations have produced multiple tests, each using a different test method. Test protocols have varied not only because test goals and available data are different from one test to the next, but also because guidelines had not existed for protocol creation.

Thesis also compares the performance of the optical and capacitive sensor in different scenarios for which protocols are written. Another objective of this thesis is to model these performance characteristics so that the output can be predicted beforehand. Then the recognition algorithm can be augmented with the modeled parameters and the threshold is changed accordingly. 


\section{CHAPTER 2 BACKGROUND WORK}

\subsection{Types of Testing}

This section summarizes the current understanding and work done by the biometrics community toward the end of field performance estimation. Although not much work has been done in the field of biometric performance testing, Best Practices in Testing and Reporting Performance of Biometric Devices [9] [20] [35] presents the best way to test and evaluate the system and this document tries to fix the variability and contradictions among the available test protocols. There are three types of evaluation that can be performed on a biometric system as defined in the introduction chapter.

Though an active area of research for past few years, testing and evaluation of biometric system is not well understood because of the following reasons:

- $\quad$ Available testing paradigms are controversial [31]:

o Open set Vs Closed set testing (Fig 2.1) 


\begin{tabular}{|l|l|}
\hline \multicolumn{1}{|c|}{ Open-Set Testing } & \multicolumn{1}{c|}{ Closed-Set Testing } \\
\hline $\begin{array}{l}\text { Do I know you? } \\
\text { Who are you? } \\
\text { (Unknown impostor) }\end{array}$ & $\begin{array}{l}\text { I know you. } \\
\text { Who are you? }\end{array}$ \\
\hline Threshold/rank dependent & Threshold independent \\
\hline $\begin{array}{l}\text { False match rates (FMR) and false } \\
\text { non-match rates (FNMR) } \\
\text { Receiver Operator Curve (ROC) } \\
\begin{array}{l}\text { Detection Error Trade-off Curve } \\
\text { (DET) }\end{array}\end{array}$ & Rank reporting \\
\hline $\begin{array}{l}\text { Consistent estimator (converges with } \\
\text { test size) }\end{array}$ & $\begin{array}{l}\text { Inconsistent estimator (does not } \\
\text { converge with test size) }\end{array}$ \\
\hline Generate histograms & Do not generate histograms \\
\hline
\end{tabular}

Figure 2.1 Open set Vs Closed set testing [31]

o Technology, scenario and operational testing: Comparison of all the three testing schemes is provided in Fig 2.2 


\begin{tabular}{|c|c|c|c|}
\hline Type of Test & Technology (in vitro) & Scenario (in situ) & Operational (in vivo) \\
\hline Database & $\begin{array}{l}\text { Typically pre-collected, usually for } \\
\text { testing multiple components }\end{array}$ & Gathered with system under test & Gathered with system under test \\
\hline Data Comparisons & Offline & Online and/or Offline & $\begin{array}{l}\text { Online (may have offline } \\
\text { component) }\end{array}$ \\
\hline Object of Testing & $\begin{array}{c}\text { Biometric component (e.g., algorithm } \\
\text { or sensor) }\end{array}$ & Biometric system & Biometric system \\
\hline Physical Environment & $\begin{array}{c}\text { Controlled or uncontrolled when } \\
\text { biometric data recorded, Not applicable } \\
\text { during testing }\end{array}$ & Controlled and/or recorded & $\begin{array}{l}\text { Not controlled, preferably } \\
\text { recorded }\end{array}$ \\
\hline User Interaction & $\begin{array}{l}\text { Maybe recorded when biometric data } \\
\text { recorded, Not applicable during testing }\end{array}$ & Recorded & $\begin{array}{l}\text { Recorded during enrollment, } \\
\text { Maybe recorded during } \\
\text { verification/identification }\end{array}$ \\
\hline User Behavior & $\begin{array}{c}\text { Controlled and/or Uncontrolled when } \\
\text { biometric data recorded, Not applicable } \\
\text { during testing }\end{array}$ & Controlled & Uncontrolled \\
\hline Results & Internally consistent & $\begin{array}{l}\text { Compromise between internal } \\
\text { and external consistency }\end{array}$ & Externally consistent \\
\hline Repeatability of Results & Repeatable (database fixed) & $\begin{array}{l}\text { Quasi-repeatable (if test scenario } \\
\text { and population controlled) }\end{array}$ & Non-repeatable \\
\hline Typical Results Reported & $\begin{array}{l}\text { Comparison of biometric components } \\
\text { or versions of components } \\
\text { (e.g., algorithms or sensors) } \\
\text { Determine critical performance factors }\end{array}$ & $\begin{array}{c}\text { Compare biometric systems } \\
\text { Determine critical performance } \\
\text { factors } \\
\text { Predict simulated performance }\end{array}$ & $\begin{array}{l}\text { Measure performance in an } \\
\text { operational environment }\end{array}$ \\
\hline Constraints & $\begin{array}{l}\text { Appropriate test database, e.g., } \\
\text { gathered with a universal sensor }\end{array}$ & Operational, instrumented system & $\begin{array}{c}\text { Operational, instrumented system } \\
\text { ( typically only decisions } \\
\text { available, scores preferable) }\end{array}$ \\
\hline Human Test Population & Recorded & Live & Live \\
\hline
\end{tabular}

\section{Figure 2.2 Testing technologies comparison [31]}

- $\quad$ The statistical models are complicated by the intricacies of real world [31]:

o Many statistical approaches assume iid (independent, identically distributed) data samples but these assumptions are not always valid.

o Correct sample size and confidence intervals determined only after data acquisition and analysis.

o Every person has their own error rates. This undermines large-scale estimates, reduces our ability to assess confidence intervals, and prevents us from answering the question "How large should the test sample be?" Recent 
large scale tests indicated that Doddington's zoo [4] is alive and well and biting.

- The performance prediction models are not available:

o The performance of a biometric system is influenced by many factors that include human behavior, environment and application.

o We don't have predictive theories to model these factors.

o We can't predict result analytically.

- $\quad$ Lack of testing protocols:

o Currently there are no scientifically based general biometric test protocols that ensure valid test results because of too many variables involved.

- Hard to get representative data

- Because of humans involved in the technology it's difficult to adapt the input variations.

- Vendors are sensitive to test result: Vendor's don't want to know the result because they want to live in the illusion of good performance.

- $\quad$ Testing costs a lot in terms of time, people, money and resources.

\section{2 existing test methodologies}

Generation of matching scores: For testing biometric system images [20] [29] are collected for template generation and eventually matching is performed for performance evaluation. There are possibly two ways of match scores generation:

- Online: Matching scores are said to be online if matching is done at the same time the image is collected. The advantage of online matching is that it saves the image storage space because images can be discarded after matching. 
- Offline: Matching scores are said to be offline when the images are collected earlier. Offline testing can be performed to give better results as full cross comparisons can be done quite easily in which each sample is compared to every other template.

Evaluation Planning: The goal of the evaluation and the type of evaluation are two major decisions that are the basis of test protocol development.

Controlling factors that influence the performance [20]: Planning a biometric evaluation performance requires the knowledge of the factors that influence the performance of the system. The best practices document divides these factors in four categories:

- Factors that are deliberately incorporated in the system to see their effect on the performance.

- Factors that are kept constant throughout the experiment.

- Factors that are ignored during the experiment.

- Factors "randomized out" of the experiment.

Volunteer selection [20]: The best practices document uses images that come from original test population and does not use artificially generated images. The volunteers selected represent the demographics that reflect the target application for which the system is being evaluated. Necessary steps were taken to circumvent the possibility of user getting bored because it may lead to user becoming less careful and hence can severely impact the performance evaluation of the biometric system. Enrollment and matching is carried out in different sessions 
Test Size: Best practices [20] recommends that the test size should be as large as practicable and number of volunteers matters more than the number of attempts in order to correctly evaluate the performance of the biometric system. Then sufficient numbers of samples per user are collected so that the total number of attempts exceeds that required by the Rule of 3 or Rule of 30 as appropriate:

- Rule of 3: It gives the lower bound on number of users or attempts needed to get a particular confidence interval. As described by the best practices document it addresses the question "What is the lowest error rate that can be statistically established with a given number $N$ of (independent identically distributed) comparisons?"

- Rule of 30: This was proposed by Doddington to determine the number of users required to attain a particular level of accuracy. It says that there should at least be 30 errors and only then we can be $90 \%$ sure that the error rate is within $30 \%$ of the observed error rate.

For these rules to be valid it is assumed that the attempts are independent and identically distributed.

\section{Data Collection:}

Data collection errors: The best practices document illustrates two types of errors that can happen during data collection process. These are corpus errors and database errors. Here corpus refers to the captured biometric image and database refers to the information 
about the corpus. The care must be taken to avoid or circumvent both the types of errors because otherwise they may lead to severe evaluation errors.

Data and details collected: The best practices document recommends saving sample images and features and to automatically log enrolments and transactions which includes all the matching scores. The collection of images can help in generating matching scores offline which avoids the unfathomable errors induced during online testing due to negligence. Moreover it will also help in evaluating different algorithms, otherwise to evaluate each algorithm separate data collection process has to be performed which is quite a cumbersome process. Also it is easy to visualize and track errors using images rather than by features only. Before start testing with real user a dry run or practice testing should be done.

For scenario testing the biometric data should be collected in a simulated environment which replicates or models the real world situation. Care must be taken to prevent any error induced by circumstances surrounding the collection. The document recommends the minimization of keystroke entry on the part of both volunteers and experimenters.

\subsection{Statistical Analysis}

Statistical methods for evaluating the confidence interval for the error rate [26]: Confidence interval is the best way to estimate a parameter. In case of biometric systems confidence interval is used to estimate the error rates. There are several methodologies [26] [33] available which have their own pros and cons. The few famous methods are by Doddington et al. (2000), Mansfield and Wayman (2002), Bolle et al. (2002), Schuckers 
(2003b), Schuckers (2003a) and Michaels and Boult (2001) and most of these methods consider $95 \%$ confidence interval.

Notation:

$$
\begin{array}{ll}
n & \text { number of enrolled volunteers } \\
m_{i} & \text { number of samples from the } i^{\text {th }} \text { volunteer }
\end{array}
$$$$
\bar{m}=\frac{\sum_{i=1}^{n} m_{i}}{n} \text { average no. of samples per volunteer }
$$

$$
x_{i j}=\left\{\begin{array}{ll}
0 & \text { Error for the } \mathrm{j}^{\text {th }} \text { attempt by the } \mathrm{i}^{\text {th }} \text { individual } \\
1 & \text { Error for the } \mathrm{j}^{\text {th }} \text { attempt by the } \mathrm{i}^{\text {th }} \text { individual }
\end{array}\right\}
$$

$$
\mathrm{X}_{\mathrm{i}}=\sum_{j=1}^{m i} x_{i j}
$$

$$
\mathbf{X}=\left(X_{1}, X_{2}, \ldots \ldots \ldots \ldots, X_{n}\right)^{\mathbf{T}}
$$

$\pi$ overall error rate

$\rho$ intra- individual correlation

$$
Z_{.025}=1.96\left(97.5^{\text {th }} \text { percentile of a Normal distribution }\right)
$$

$$
\begin{aligned}
& \hat{\pi}=\frac{\sum_{i=1}^{n} X_{i}}{\sum_{i=1}^{n} m_{i}} \\
& p_{i}=\frac{X_{i}}{n} \text { proportional error commited by each individual }
\end{aligned}
$$

The methods listed above are used to estimate variability in this error rate. They have different approaches towards this estimation. 


\section{Doddington's Rule:}

This approach is based on binomial distribution and is used when

$$
\sum_{i=1}^{n} X_{i} \geq 30
$$

The confidence interval is created by taking the estimated error rate and adding and subtracting $30 \%$ of that estimated error rate.

\section{Best Practices:}

This approach is used in the "Best Practices" document and this methodology takes $\hat{\pi}$ as the point estimate of $\pi$. The confidence interval is created by taking the estimated error rate and adding and subtracting a quantile of the normal distribution to a standard error based on the variability of the individual error rates. The confidence interval is given by

$$
\hat{\pi} \pm 1.96 \sqrt{\frac{\sum_{i=1}^{n}\left(m_{i}\left(p_{i}-\hat{\pi}\right)\right)^{2}}{\bar{m}^{2} n(n-1)}}
$$

where the term in the square root is the estimated variance of the observed error rate.

\section{Subset Bootstrap method:}

Often for the confidence interval [27] [3] [5] estimated by the best practices approach gives the negative result which is not possible. This is because observed error rate has a non normal distribution. A method given by Bolle that uses non- parametric approach rectifies this problem and is known as "subset bootstrap". This approach does not make any assumption related to underlying distribution and the dependencies between attempts. For error estimation it uses the bootstrap resampling approach.

\section{Beta Binomial:}


This technique [28] was proposed by Schuckers and uses MLE (Maximum Likelihood Estimation) for interval estimation. This is a parametric technique unlike the above technique which is non parametric in nature. Author modeled the mean and variance of the estimate and estimates the parameters of the model. The model is as follows:

$$
\begin{gathered}
E\left[X_{i}\right]=m_{i} \pi \\
\operatorname{Var}\left[X_{i}\right]=m_{i} \pi(1-\pi)\left(1+\left(m_{i}-1\right) \rho\right)
\end{gathered}
$$

One of the assumptions made by Schuckers is that all the $X_{i}$ 's are conditionally independent. The Best Practices and Beta binomial approach performs nearly identical.

Logit Beta-binomial: This technique is again a parametric approach and is same as above with the only difference that it creates a confidence interval for $\log i t(\pi)$ instead of $\pi$ and then transform the interval back to $(0,1)$ interval. The confidence interval for $\log i t(\pi)$ is given by

$$
\log \operatorname{it}(\hat{\pi}) \pm 1.96 \sqrt{\frac{1+(\bar{m}-1) \hat{\rho}}{\hat{\pi}(1-\hat{\pi}) \bar{m} n}}
$$

which is again transformed back to the inverse $\log i t(\pi)$ to get interval in the range $(0,1)$. This approach gives the best results i.e. it gives best coverage area even for smaller values of $m$ and $n$.

\subsection{Evaluation of fingerprint readers in different scenarios}

There is a recent study by a group of Korean researchers [17] headed by Dr. Hale Kim about the effect of environmental and human factors on fingerprint readers. The purpose of the study is to compare the characteristics of fingerprint readers and to provide a framework of performance evaluation of various sensing mechanism. They measure the 
quality of fingerprint image because it indicates the performance of fingerprint readers according to changes in user behavior and environment. Here are some of the results Fig 2.3 and 2.4 provided by them:

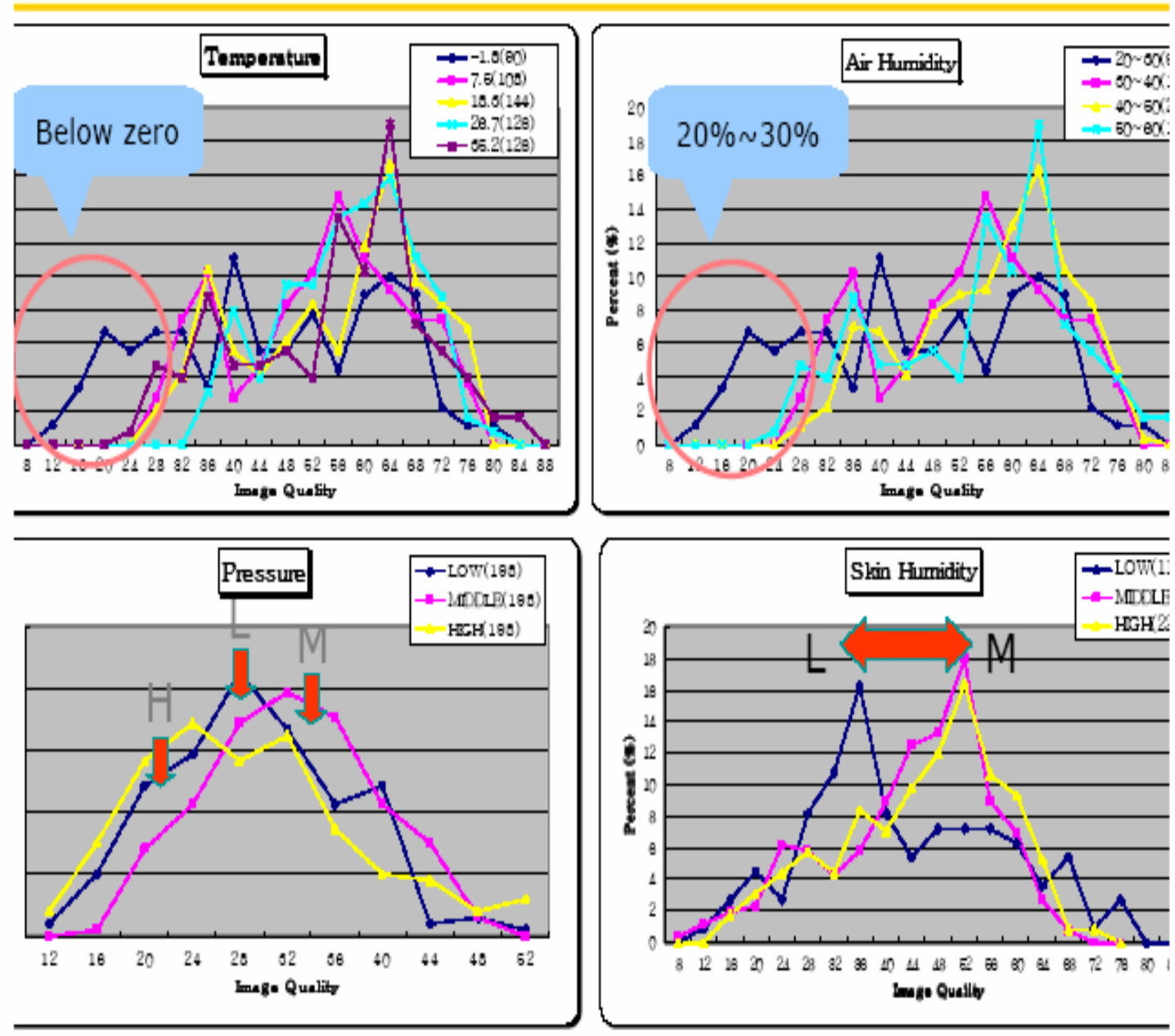

Figure 2.3 Effect of Environmental and Human factors

on optical fingerprint sensor[17] 


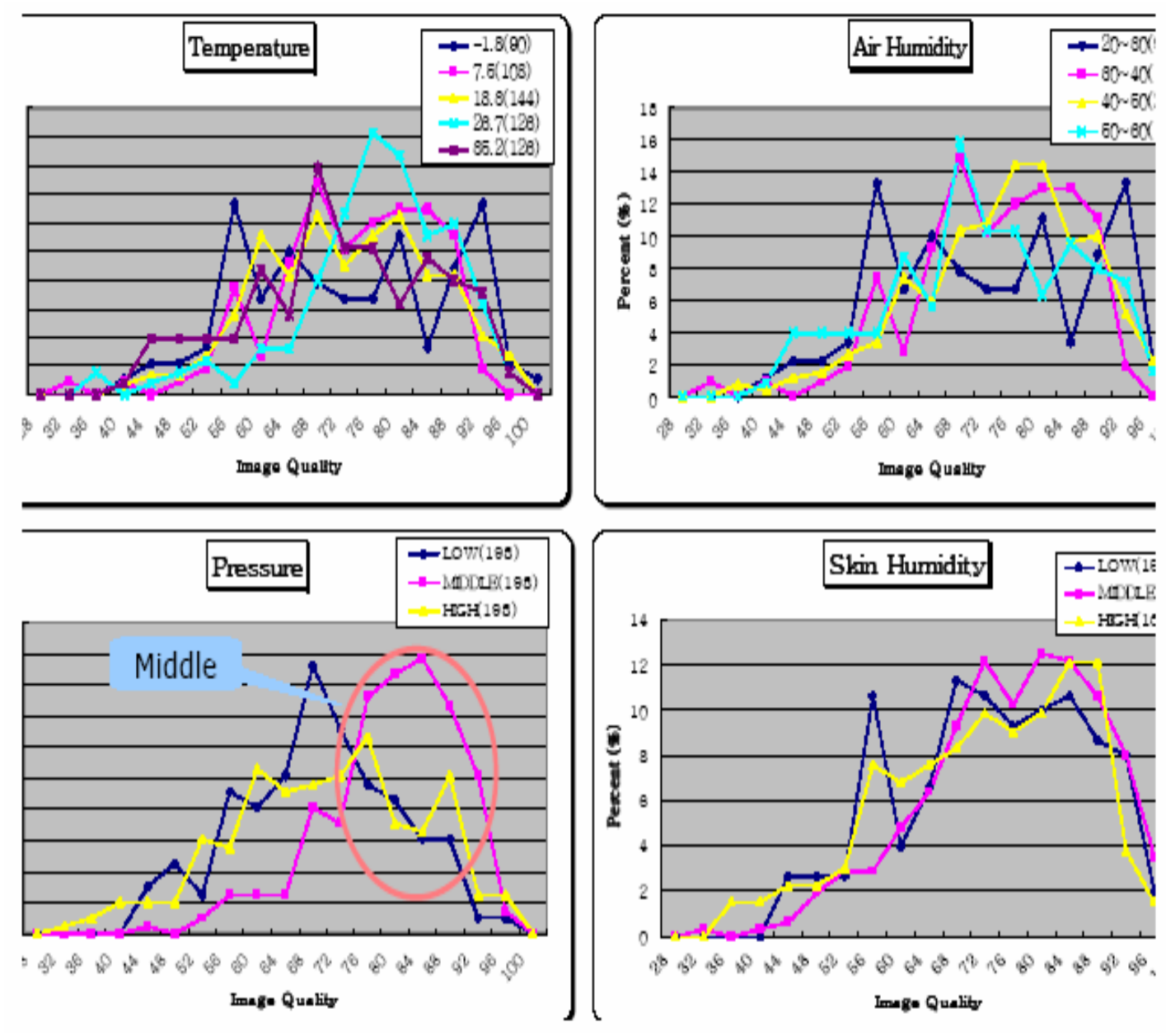

Figure 2.4 Effect of Environmental and Human factors

on Semiconductor fingerprint sensor [17]

The graphs presented shows the affect of environmental and human factors like temperature, air humidity, pressure, skin humidity on image quality distribution generated from optical and capacitive fingerprint sensor. The different color line shows the value of the considered factor. For example in Fig 2.4 we can see the affect of different temperature values on the image quality distribution. 
As can be seen from the graphs the quality of the fingerprint for optical sensors decreases when the temperature decreases due to dryness of skin but does not change for capacitive sensor.

As far as human factors are concerned the images captured from the optical sensor gets smaller for the low pressure but with capacitive sensor images are good not only with the middle pressure but also with the high pressure.

Skin humidity also affects negatively to the optical sensor unlike the capacitive sensor which proves to be quite robust against skin humidity. 


\section{CHAPTER 3 GENERIC TEST PROTOCOLS}

\subsection{Generic modes of operation}

Over the past few years, many documents have been published addressing the issue of biometric device testing [23], but few have attempted to develop generic testing protocols for biometric systems. Such a document describing generic test protocols for biometric systems is needed because there are many contradictory and conflicting test protocols for biometrics devices published. This will help standardize testing efforts, by making testing more generic and by taking the entire biometric system into account, as opposed to just the biometric device.

The first step is to decompose a biometric system into various generic components and applications.

A biometric system can viewed as being either a stand-alone system or a networked system. Next, the system can either control physical access or logical access. The next crucial component is to evaluate which operational modes are possible. Some possible modes are:

- Acquisition: The process of acquiring the biometric data from the user is known as acquisition. The output parameter (performance parameter) that will be affected by this is FTA (Failure to acquire). As shown in Fig 3.1, the acquisition mode's input is the biometric trait(s) and its output is the raw image(s) of the trait. The biometric trait(s) may need to be re-imaged if the initial image(s) do not pass the quality control parameter. The quality of raw 
images will greatly affect other related modes. Poor image quality will create a snowball effect throughout the system.

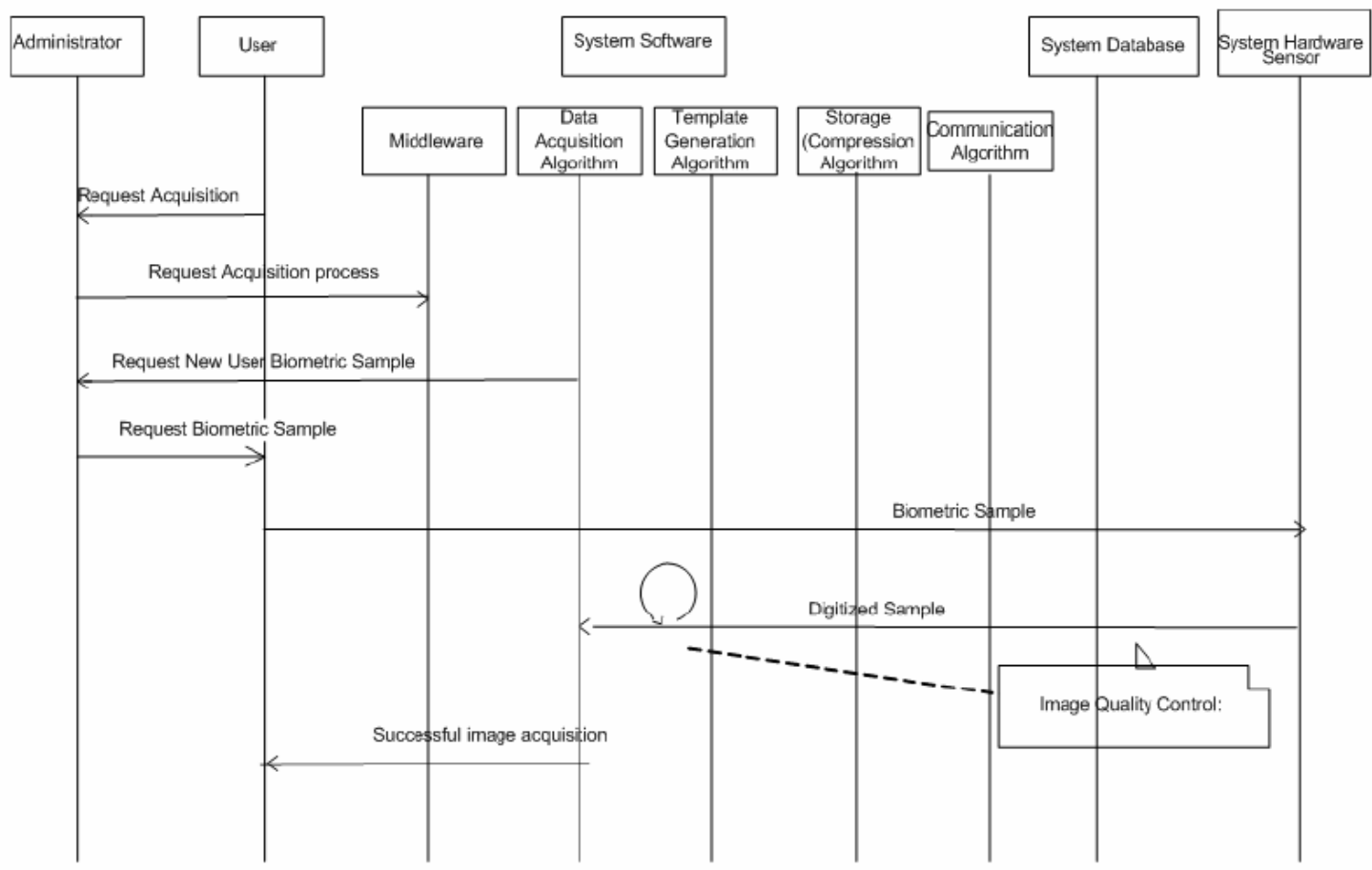

Figure 3.1 Acquisition Mode [23]

- Enrollment: The process of collecting biometric samples from a person and the subsequent preparation and storage of biometric reference templates representing that person's identity. FTE (Failure to enroll) is the performance parameter that will be affected by this mode. Inputs to the enrollment mode are results from the acquisition mode, the algorithm to use to generate templates, and user specific parameters. Next, a query is done to determine if the user already exists in the user database. Then the user template is generated and quality score is computed. The quality of templates will greatly 
affect other related operational modes. Poor template quality will propagate throughout the system, diminishing performance. See Fig 3.2 for a representation.

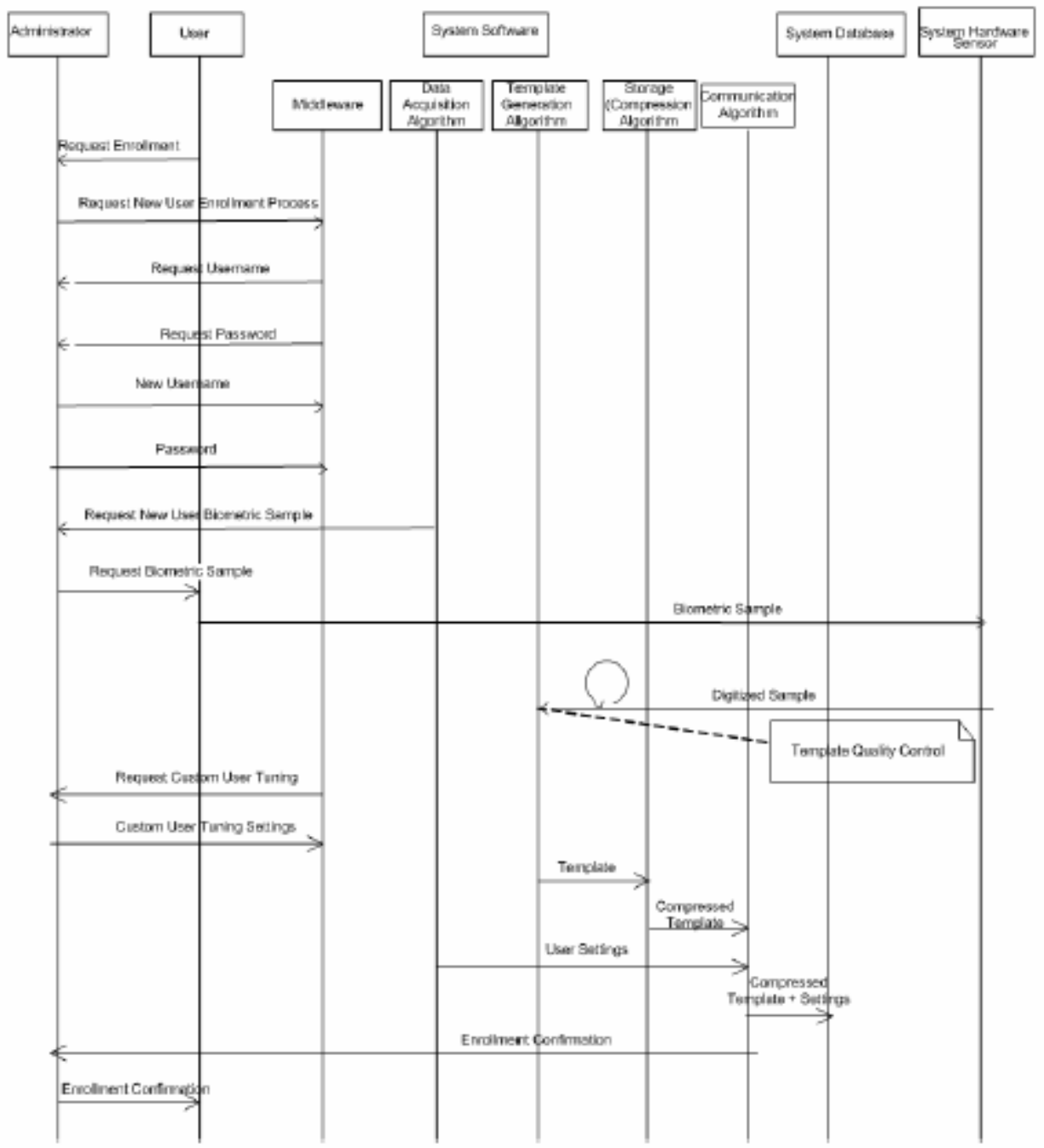

Figure 3.2 Enrollment Mode [23]

- Verification: A comparison of two sets of biometrics to determine if they are from the same individual; or, in fraud prevention applications, a one-to-one comparison of a live finger and a previously enrolled record to ensure that the 
applicant is who he/she claims to be. This mode will affect V_FRR (Verification False Reject Rate) and V_FAR (Verification False Accept Rate). Inputs to the verification mode are user login information, results from image acquisition, template generation and matching algorithm, and user specific parameters. As shown in Fig. 3.3, the intermediate steps are to generate the user template for matching and then perform a one to one matching on the user database. The results of the verification mode will be a matching score upon which a decision is made.

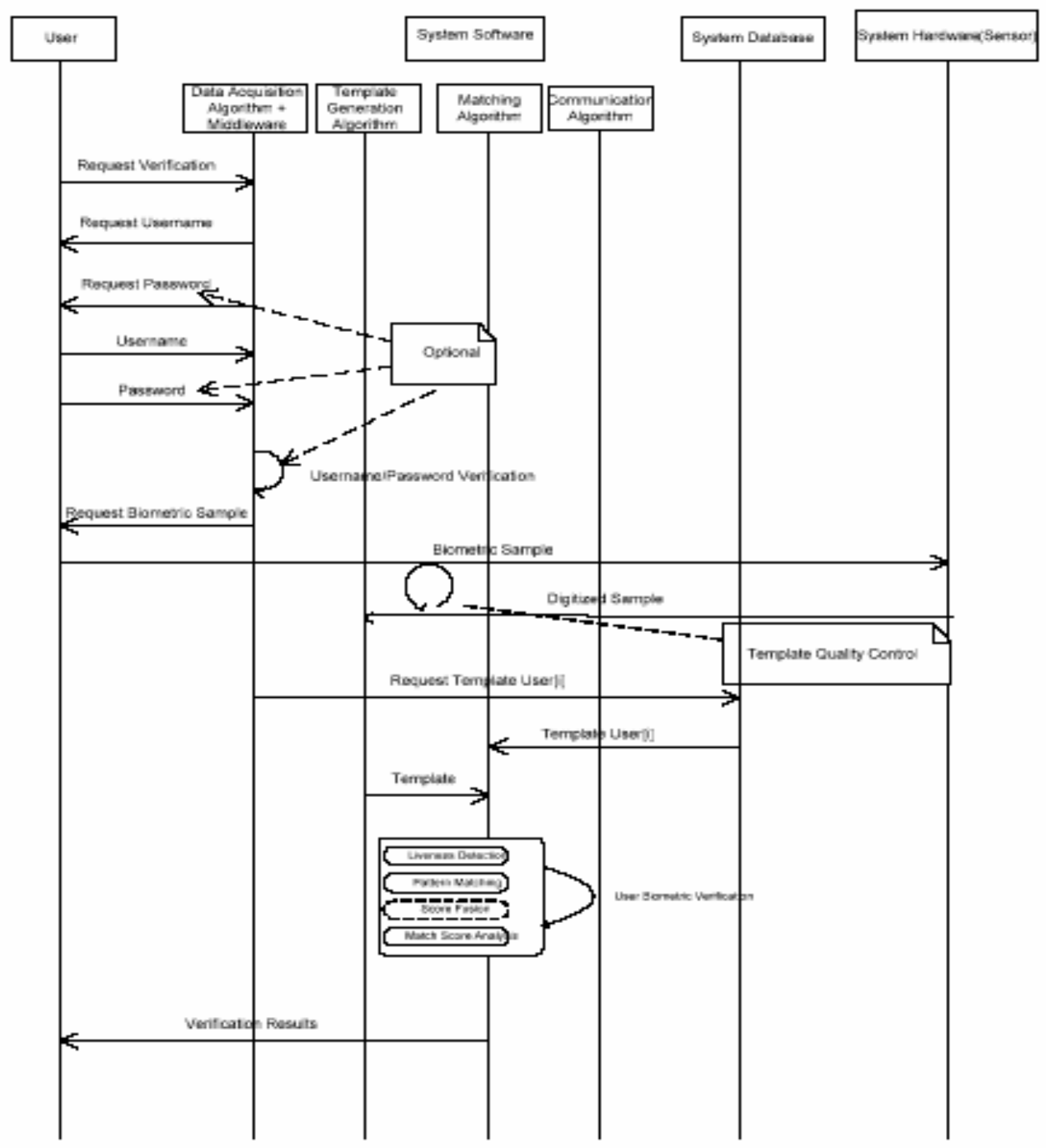




\section{Figure 3.3 Verification Mode [23]}

- Identification: A one-to-many comparison of an individual's submitted biometric sample against the entire database of biometric reference templates to determine whether it matches any of the templates and, if so, the identity of the enrollee whose template was matched. The biometric system using the one-to-many approach is seeking to find an identity within a database, rather than verify a claimed identity (Contrast with verification). This mode will affect I_FRR (Identification False Reject Rate) and I_FAR (Identification False Accept Rate). Inputs to the identification mode are matching algorithm, results from image acquisition, and template generation algorithm. As shown in Fig. 3.4, the intermediate steps are to generate the user template for matching and then perform a one to one matching on the user database. The results of the identification mode will be a matching score upon which a decision is made. 


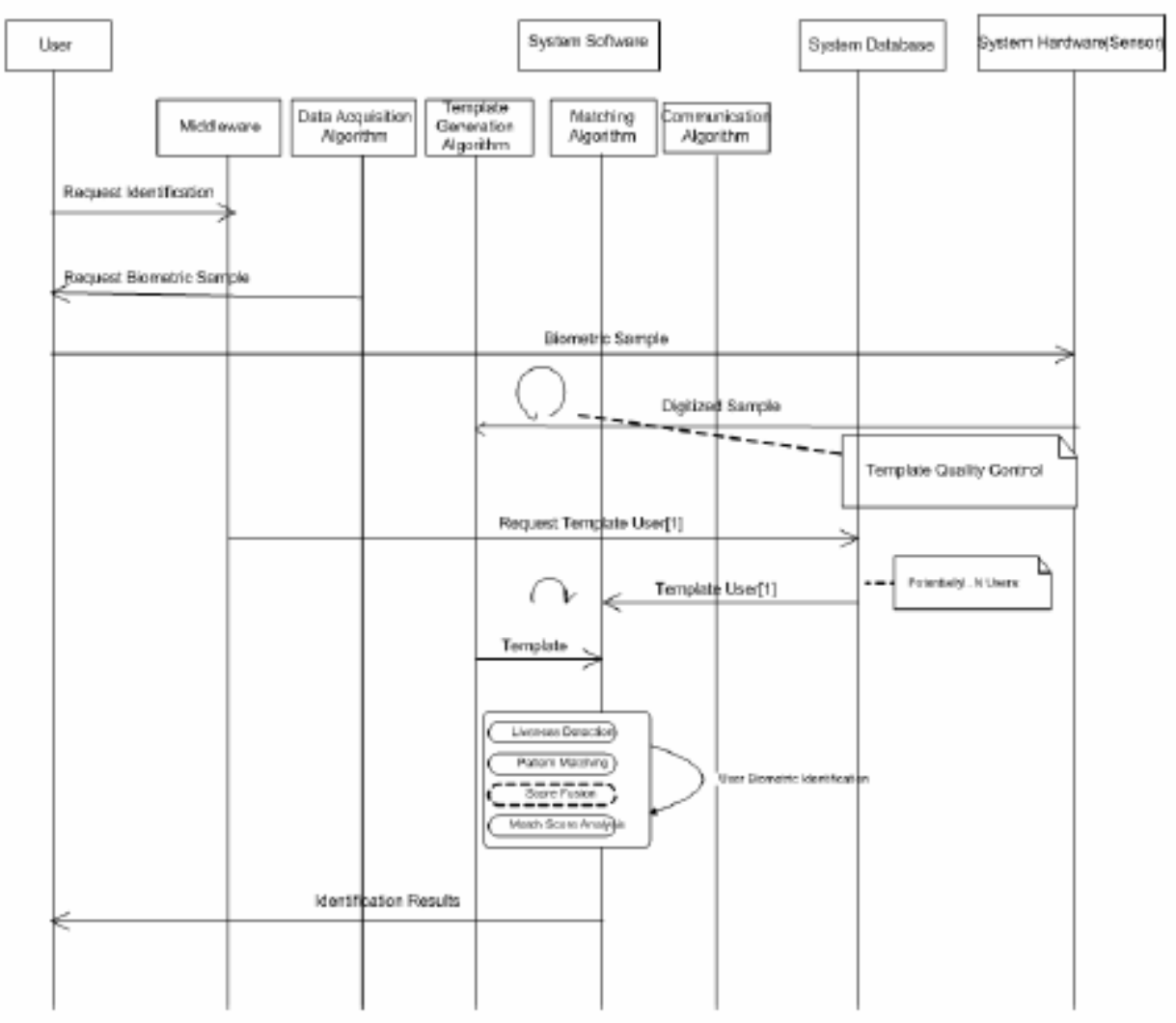

Figure 3.4 Identification Mode [23]

- User Template update: User template update is the process of updating a user's templates stored in the system to counteract template aging. FTE will be affected by this mode. Inputs to the user template update mode are results from the acquisition mode, the algorithm to use to generate templates, and user specific parameters. Next, a query is done to retrieve the user record from the user database. Then the user template is generated and quality score is computed. User templates can vary over time and it is necessary to periodically update user templates to combat template aging. The 
performance of the biometric system will be greatly affected by how current the templates are in the user database. See Fig. 3.5 for a representation.

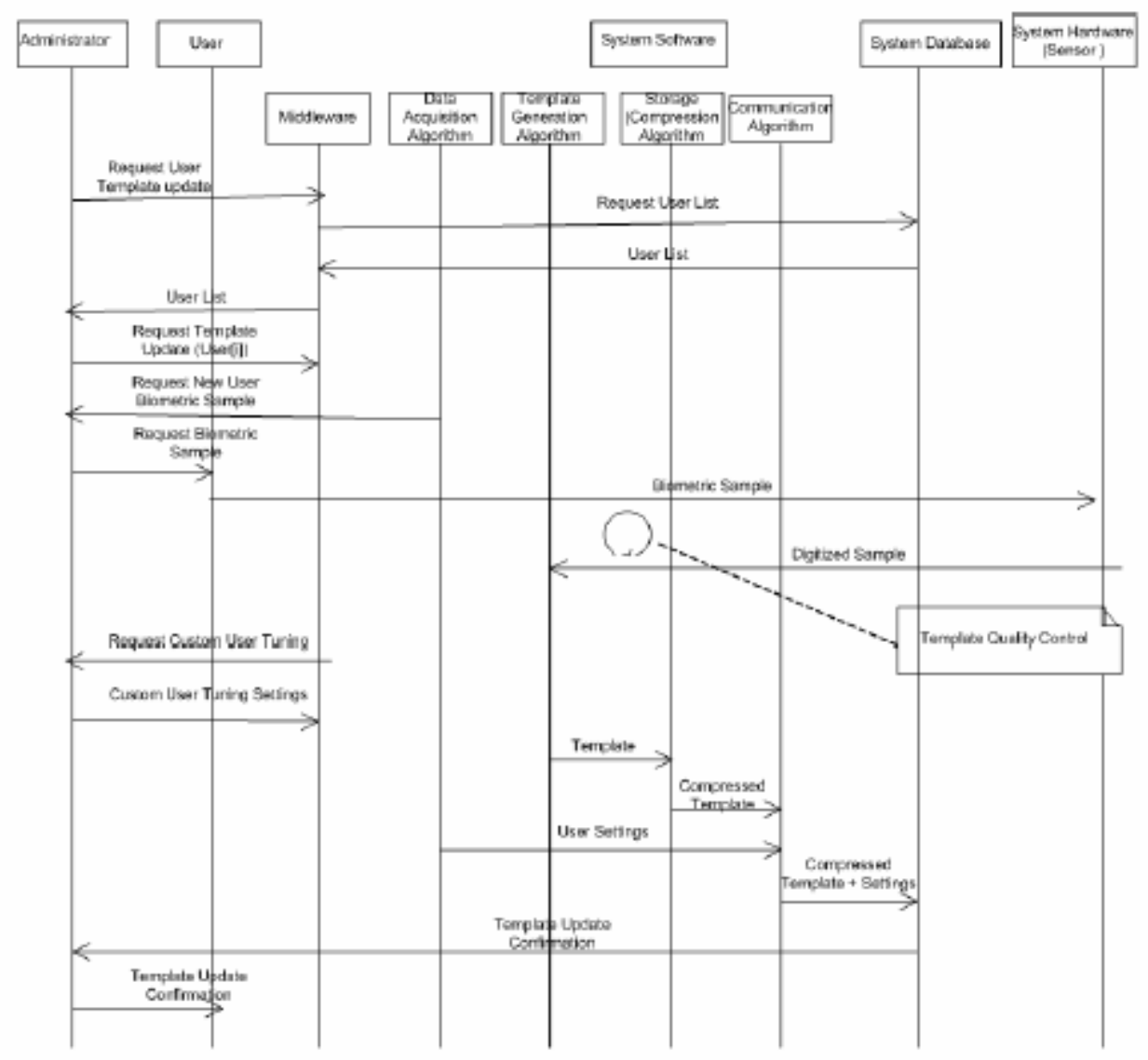

Figure 3.5 User Template Update Mode [23]

\subsection{Variables impacting the generic modes of operation}

In this thesis I present scenarios that are independent of the modality used and are therefore relevant to any modality (ex. fingerprint, hand geometry, iris, face, etc.).

Now that we have considered several possible operational modes for a generic biometric system, we must now determine the scenarios in which the biometric system can be tested. The key to these testing scenarios are that they must be repeatable and generic. It is very important for testing to be repeatable because it will allow the ability for testing 
results to be used as a standard means for comparing biometric systems. However, repeatability in biometric testing is a very difficult task because all of the environmental variables must be accounted for and controlled to some degree and even then there will still some variability. The goal of this research is to help develop testing protocols that can greatly improve the repeatability of testing protocols that are not currently available.

It is important that these test protocols and scenarios also be generic so that they can be applied to any biometric system. This will greatly aid in the possible standardization efforts in biometric system testing. Once the main testing scenarios have been identified, they can be elaborated down the road to further test a specific biometric system per user basis.

Some of the main scenarios that have been considered are:

- Sensor cleaning frequency: How often the device should be cleaned? Cleansing of the device will improve the system performance as it will lead to quality image capture. Testing can be done to evaluate the system's performance to variations in the periodicity of cleaning.

- Indoor and outdoor: The indoor and outdoor is an important scenario to test as according to applications device can be installed anywhere. Testing can be done to evaluate the system's performance to change in environmental temperature condition.

- Device placement: The device placement considers the effects of placing your biometric device at different positions. It is the place where your biometric device is placed. The key factor to consider is whether the device during enrollment is at the same location relative to the user as the device during the 
image acquisition process for other operational modes. Location can be tested by comparing the performance of the device when location is varied to when it is held constant. Variations in location can result from changes in height, altitude, angle, and surface of the device placement. There are two angles to consider: $\sigma$ and $\Phi$. Location is an important factor because if it varies through out the system's deployment then results can greatly be altered because of its effects on biometric presentation which greatly affects image acquisition quality.

- Biometric presentation (pose and/or orientation): Biometric presentation considers the effects of the way a user presents their biometric trait(s) to the system. The presentation of a user's biometric trait(s) greatly affects the system's ability to correctly match/identify genuine users. This can be subdivided into a) the angle of rotation and b) distance from the device. One approach to evaluating a system's susceptibility to variations in pose and/or orientation would be to compare the system's results for various poses and orientations. Image quality will be affected which will eventually affect the biometric system's performance in matching templates. 


\section{CHAPTER 4 EXPERIMENTS}

\subsection{Protocols}

In the last chapter I have identified the variables that can affect the performance of the biometric system. In my thesis apart from coming up with the variables affecting the modes of operation I have come up with the test protocols for the testing few of these variables.

These protocols have been written to reduce the errors induced during the testing of the fingerprint recognition system. The protocols described later are written to test these variables so that we can compare the performance of optical and capacitive sensors:

In my thesis I am comparing the performance of two sensors for the following variables:

- Sensor cleaning frequency

- Indoor and outdoor

- Device placement

- Biometric presentation

Protocols overview: Over the past few years, many documents have been published addressing the issue of biometric device technical testing, but few have actually attempted to develop the testing protocols for biometric systems. This is an effort contributing towards standardizing the testing protocols. Most important thing about testing is that they should be repeatable which is possible only if the modeled scenario can be carefully controlled. However, repeatability in biometric testing is a very difficult task because all of the environmental variables must be accounted for and controlled to some degree and even then there will still some variability. The goal of this thesis is to 
help develop testing protocols that can greatly improve the repeatability of testing protocols. In this research I have tried to generate the testing protocols for fingerprint sensor with respect to change in sensor cleaning frequency, environment(when the device is moved from indoor to outdoor)and the way user presents their biometric(rotation and translation).

- Assumptions: Throughout the test it was assumed that the scenario was one of positive verification with co-operative non-habituated and overt systems. The users are assumed to have healthy biometrics and they represent all age groups, demographics and both of the genders. The environmental factors like humidity, temperature and lighting should be simulated "normal operating conditions" as stated by the vendor and should be held as constant as possible throughout the testing.

- Environmental Variables: The environment is very difficult to control and presents a great challenge in producing repeatable results. The main environmental variables which can affect the performance of the biometric system are temperature, humidity and lighting. In my protocol I have used normal operating conditions as stated by the vendor. The room's fluorescent lighting should always be on, and the window blinds should be down to reduce effects of daylight (Lighting) variations.

- Session: To reduce any effects of template aging and any changes in user habituation, enrollment of the user and this test should occur in the same session. Same session here refers to the condition where user is supposed to 
present their fingerprints for enrollment and verification in a given short span of time.

- Biometric Presentation: Biometric presentation considers the effects of the way a user presents their biometric trait(s) to the system. The presentation of a user's biometric trait(s) greatly affects the system's ability to correctly match/identify genuine users. This can be classified as:

Rotation: This is the angle the fingerprint is presented to the sensor with respect to the horizontal plane of the sensor in degrees. To ensure that the testing is reliable and repeatable we have used the calibrated mask as shown in the Fig 4.1 and 4.2 below marked with angle $\theta$.

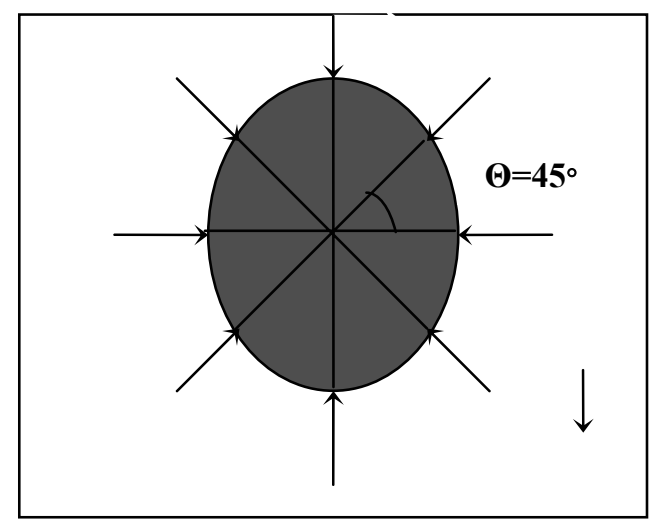

Figure 4.1 Angles of Rotation 


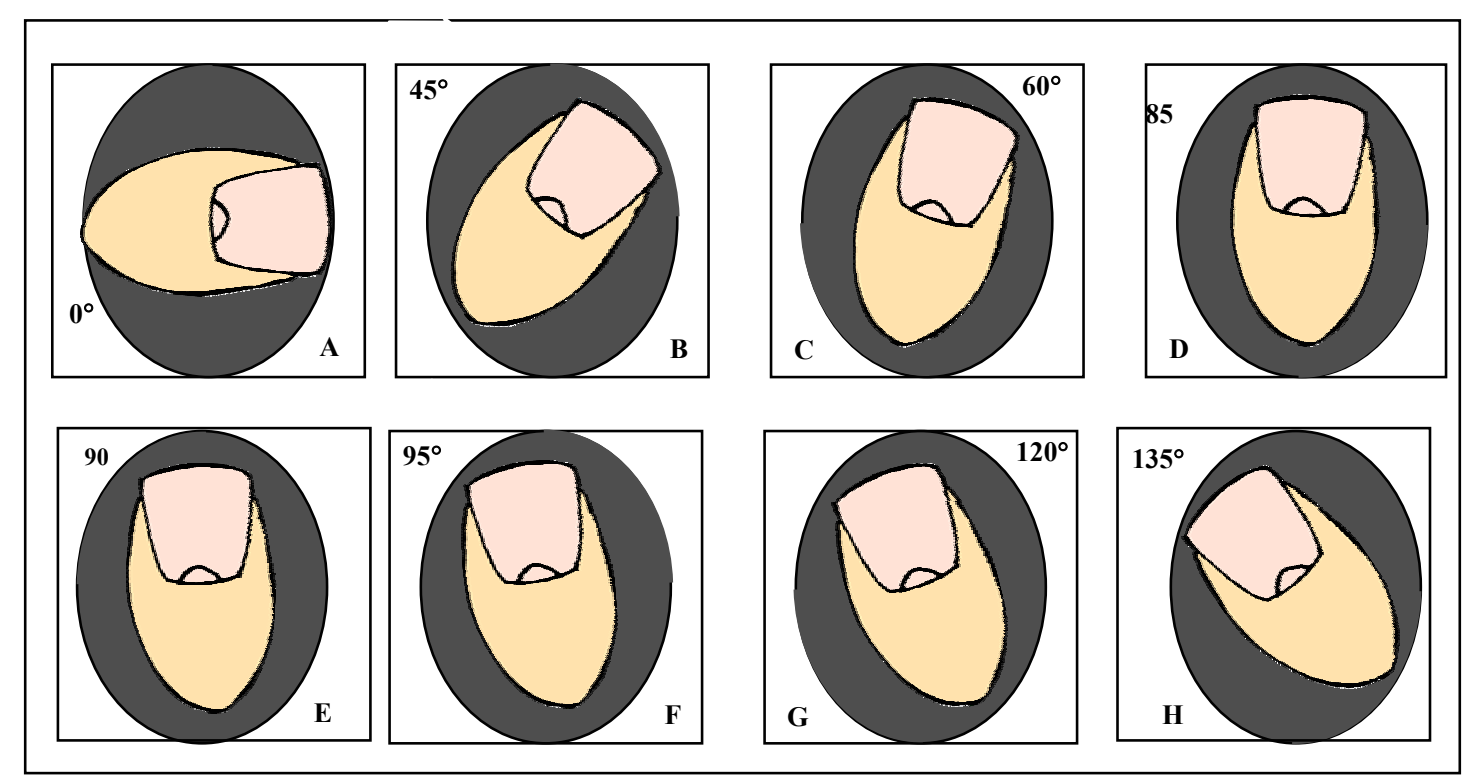

Figure 4.2 Fingerprint scanning positions.

Device Placement: The device placement considers the effects of placing your biometric device at different positions. It is the place where your biometric device is placed. The key factor to consider is whether the device during enrollment is at the same location relative to the user as the device during the image acquisition process for other operational modes. Location can be tested by comparing the performance of the device when location is varied to when it is held constant. Variations in location can result from changes in height, altitude, angle, and surface of the device placement. There are two angles to consider: $\sigma$ and $\Phi$. See Fig. 4.3 for a graphical representation. Location is an important factor because if it varies throughout the system's deployment then results can greatly be altered because of its effects on biometric presentation which greatly affects 
image acquisition quality. In my protocol I have used a robotic tripod to change the angles.

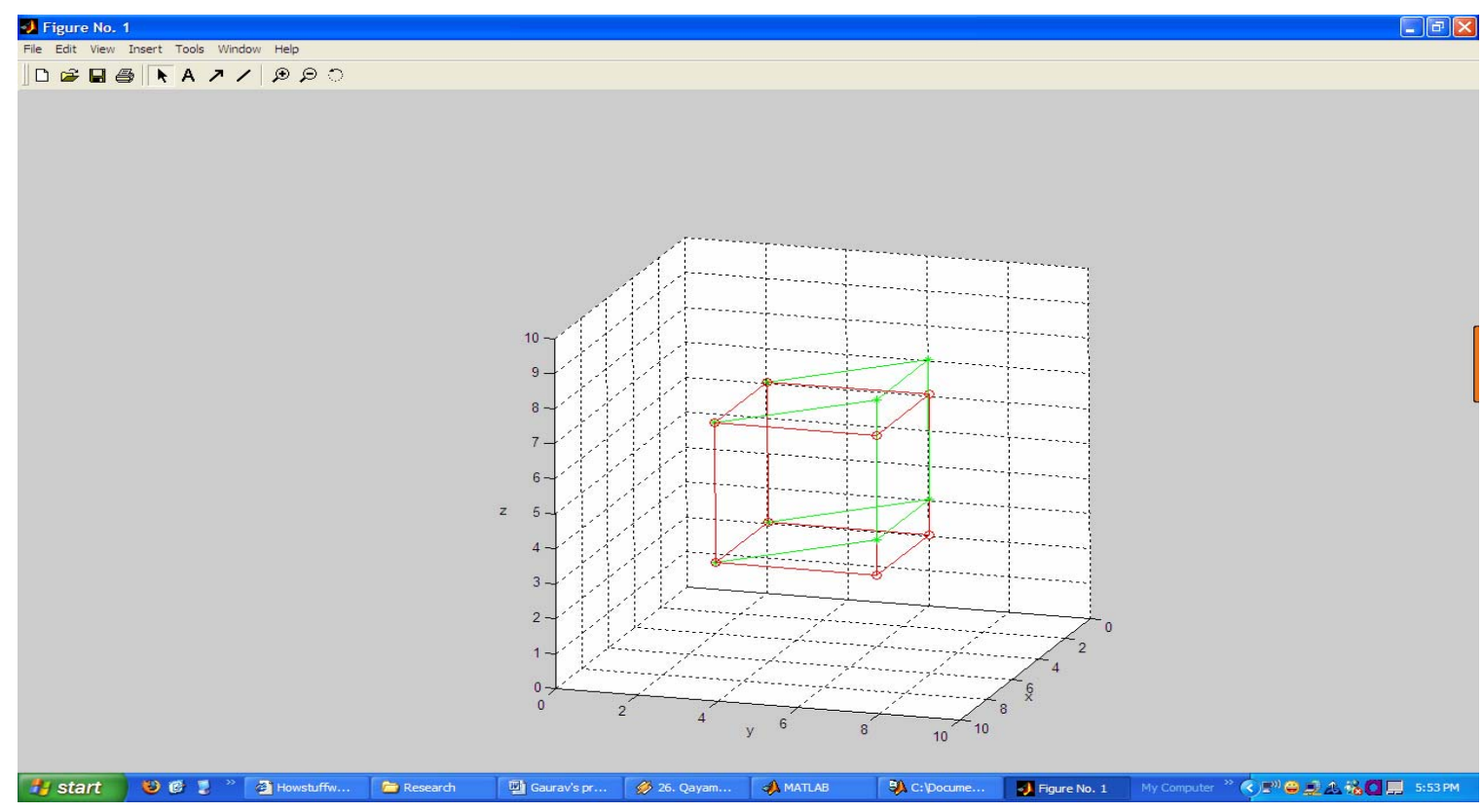

(a)

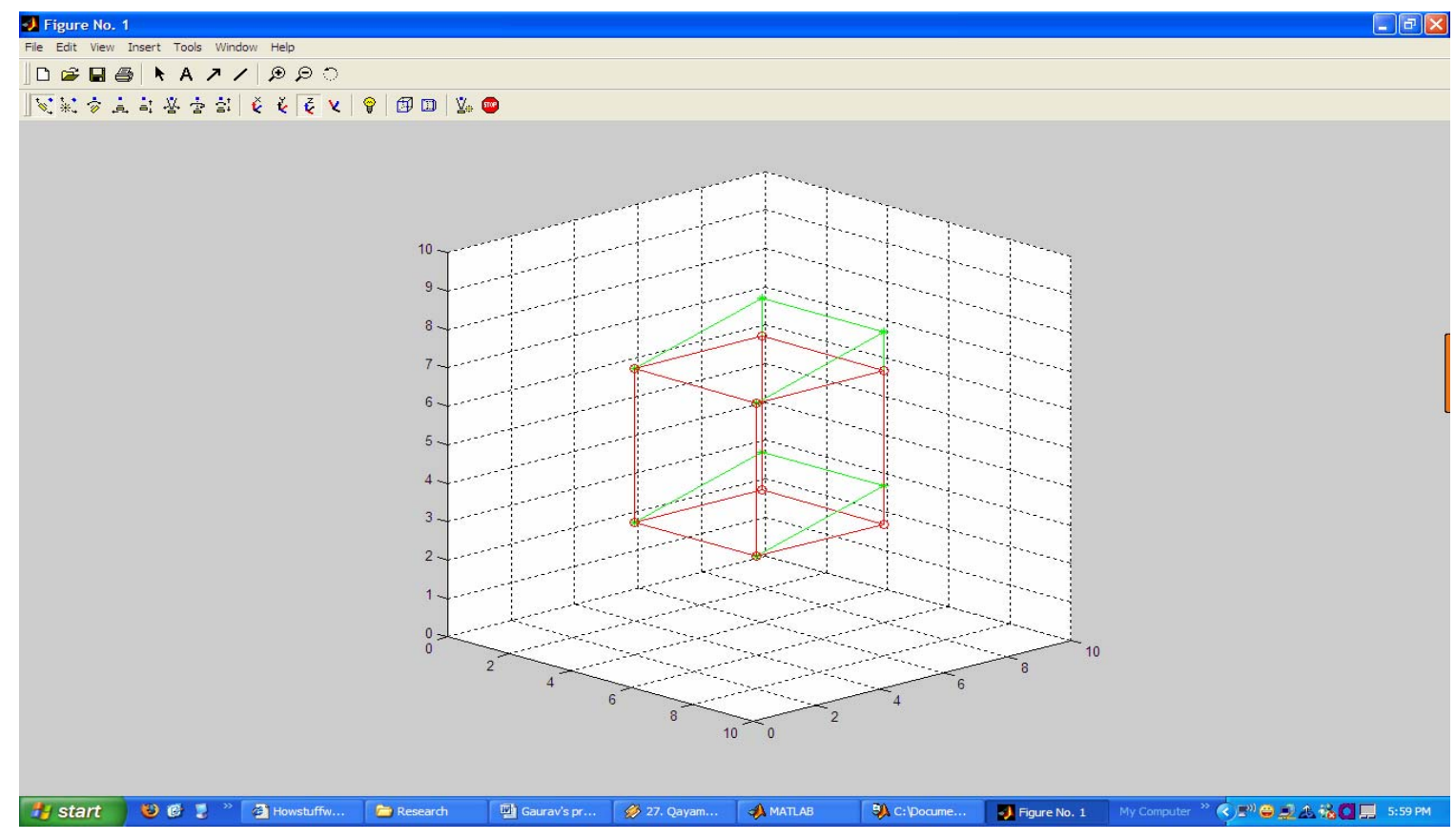

(b)

Figure 4.3 Device placement (a) Change in $\sigma$ (b) Change in $\Phi$ 
The normal way of putting the finger on sensor is by keeping angle of rotation i.e. $\theta$ equal to $90^{\circ}$ with no translation. Also the device should be placed at angles $\sigma$ and $\Phi$ both equal to $0^{0}$.

\subsection{Hardware Used}

- Secugen sensor: The optical fingerprint sensor used in the research is Secugen EyeD Hamster, model: HFDU01A (See Appendix A).

- Authentec sensor: The capacitive fingerprint sensor used in the research is Authentec.

- Temperature/Humidity Meter: A temperature and humidity meter was used to measure the test environment's temperature and relative humidity. The meter used in this testing was: Amprobe Digital Sling Psychrometer: THWD-2i. It is able to measure temperature in the range of -20 to 60 Celsius and relative humidity in the range of 1 to 99\% (See Appendix C).

- Light Meter: A light meter was used to measure luminance of the test environment in lux, which is lumen per square meter.

- Robotic Tripod: The robotic tripod is used to control the device placement variables, angles of pan and tilt. The device used it called "Tracker Pod" and the angles can be controlled through a USB port on a pc using the software included (See Appendix D).

\subsection{Software used:}


- Image acquisition software for Secugen optical sensor: This software was developed by kiran Kumar Tadaka (WVU graduate student) for CITER (Center for Identification Technology Research). This software uses .dll files provided by the Secugen I their SDK.

- Image acquisition software for Authentec capacitive sensor: This software is provided in USB Technical Evaluation Kit REV. 5.1 that comes with the Authentec sensor.

- Secugen SDK: The Software Development Kit used in this research was for the SecuGen Hamster optical fingerprint sensor. The software is named: FDx Development Kit by SecuGen.

- Data Collection software: This is the software I wrote to facilitate the data collection process. Data collection errors are the most common errors that occur while collection of biometric data. To overcome it and keep track of the testing protocol, user data and environmental variable I developed this program in JAVA.

- Verifinger Software: I have used Verifinger 4.2, a fingerprint identification algorithm developed by Neurotechnologija for matching purpose. This software is again modified by Kiran Kumar Tadaka to get the matching scores instead of a matching decision.

\subsection{Testing Protocol for frequency of sensor cleaning}

Purpose: This is a testing protocol generated for performance evaluation of fingerprint sensor with respect to change in the frequency of sensor surface cleaning. 
Environmental Settings: The environmental factors like humidity, temperature and lighting should be simulated "normal operating conditions" as stated by the vendor and should be held as constant as possible throughout the testing. The room's fluorescent lighting should always be on, and the window blinds should be down to reduce effects of daylight variations. The device should be placed in a chamber to keep control of the temperature and humidity. All these values must be properly documented as well. To reduce any effects of template aging and any changes in user familiarity, enrollment of the user and this test should occur in the same session. Also one of the obvious things is to use the same finger at both the time of enrollment and identification/verification. If the vendor does not specify the normal environmental conditions we would assume these to be:

- Standard room temperature $\left(67-72^{\circ} \mathrm{F}\right)$

- Standard humidity $(35-40 \%)$

- Standard level of lighting

Device placement and Biometric Presentation: Biometric device placement variables $(\sigma$ and $\Phi)$ should be in accordance with what is stated by the vendor. For the purpose of evaluating the performance of the fingerprint recognition system I have kept these angles as 0 degrees each. Also biometric presentation variable, angle of rotation should be at 90 degrees. The height of the device from the floor, the altitude of the device and type of the surface on which it is placed should also be documented.

- $\sigma=0^{\circ}$

- $\Phi=0^{\circ}$

- $\theta=90^{\circ}$ 
Time difference between Enrollment and Recognition: To reduce any effects of template aging and any changes in user habituation, enrollment of the user and this test should occur in the same session. Same session refers to the condition where user is 3 supposed to present their fingerprints for enrollment and verification in succession. In this manner we can ensure the same ambient conditions for enrollment and identification / verification. Also the threshold values should be set and held constant throughout the testing process.

Testing: In the above mentioned temperature and humidity controlled chamber, the fingerprint sensor and the test subject should be appropriately placed and the test subject must be able to properly present their fingerprint(s) to the device while maintaining isolation from the outside environment.

Once everything is properly installed, the enrollment is done at the normal operating conditions (as stated by the vendor) maintained in the chamber. The template is generated and stored in the database. The sensor is cleaned and the user is asked to present their biometric (fingerprint), and again a template is generated and matched against the enrolled one that was generated in the normal conditions. The same testing procedure should be followed for all users.

Now without cleaning the sensor user is asked to present their biometric (fingerprint). The template is generated and matched against the stored one that was generated in the normal conditions. The same testing procedure should be followed 100 times without cleaning the sensor. The average match scores are calculated for every five touches.

Output: The image quality score of each sample and the (FTE) Failure to Enroll rate and (FTA) Failure to Acquire rate should be documented. Also the FAR and FRR for 
identification and verification should be recorded. These performance measurements will help to determine the effects of change in sensor cleaning frequency on the fingerprint recognition system.

\subsection{Testing Protocol for indoor outdoor temperature difference}

Purpose: This is a testing protocol generated for performance evaluation of fingerprint sensor with respect to change in environment (temperature) when the system is moved from indoor to outdoor conditions. This is an effort contributing towards improvement of biometric systems. The test scenario was one of positive verification with co-operative and non-habituated users. The users are assumed to be having healthy biometrics and they represent all age groups, demographics and both of the genders.

Environmental Settings: The environmental factors like humidity, temperature and lighting should be simulated "normal operating conditions" as stated by the vendor and should be held as constant as possible throughout the enrollment process. The room's fluorescent lighting should always be on, and the window blinds should be down to reduce effects of daylight variations. The device should be placed in a chamber to keep control of the temperature and humidity for enrollment purpose. All these values must be properly documented as well. Also the sensor should be cleaned before it's every use to avoid variation in the performance due to sensor surface. To reduce any effects of template aging and any changes in user familiarity, enrollment of the user and this test should occur in the same session. Also one of the obvious things is to use the same finger at both the time of enrollment and identification/verification.

- Standard room temperature $\left(67-72^{\circ} \mathrm{F}\right)$

- Standard humidity $(35-40 \%)$ 
- Standard level of lighting

Biometric Presentation: Biometric device placement variables ( $\sigma$ and $\Phi$ ) should be in accordance with what is stated by the vendor. For the purpose of evaluating the performance of the fingerprint system I have kept these angles as 0 degrees each. Also biometric presentation variable, angle of rotation should be at 90 degrees with no translation. The height of the device from the floor, the altitude of the device and type of the surface on which it is placed should also be documented.

- $\sigma=0^{\circ}$

- $\Phi=0^{\circ}$

- $\theta=90^{\circ}$

Time difference between Enrollment and Recognition: To reduce any effects of template aging and any changes in user habituation, enrollment of the user and this test should occur in the same session. Same session here refers to the condition where user is supposed to present their fingerprints for enrollment and verification in succession. In this manner we can ensure the same ambient conditions for enrollment and identification / verification. Also the threshold values should be set and held constant throughout the testing process.

Testing: In the above mentioned temperature and humidity controlled chamber, the fingerprint sensor and the test subject should be appropriately placed and the test subject must be able to properly present their fingerprint(s) to the device while maintaining isolation from the outside environment. 
Once everything is properly installed, the enrollment is being done at the normal operating conditions as stated by the vendor maintained in the chamber. The template is then generated and stored in the database.

Now place the device at any outdoor location and all the environmental variables namely temperature, humidity and lighting are measured and documented. The user is now asked to present their biometric (fingerprint) in the outdoor settings and again a template is generated and matched against the stored template that was generated in the normal conditions (indoor). The same testing procedure should be followed for all the users.

The whole recognition process is again repeated at some other time of the day or on any other day with different environmental (temperature) conditions.

Output: The image quality score of each sample and the (FTE) Failure to Enroll rate and (FTA) Failure to Acquire rate should be documented. Also the FAR and FRR for identification and verification should be recorded. These performance measurements will help to determine the affects of change in environmental conditions on the fingerprint device.

For other two variables i.e. angle of rotation and device placement I have used protocols generated by Mr. Travis Rosiek in his thesis (Fingerprint Testing Protocols for Optical Sensors) work. 


\section{CHAPTER 5 RESULTS}

In order to strengthen the validity of my results I have used reasonable number of users. Twenty users volunteered to offer their fingerprint from four fingers (left index, right index, left middle, right middle) for the testing purpose. So that makes the total count of different fingerprints as eighty. The respective protocols are tested as mentioned in the last chapter. In order to better analyze the test results I have used different graphical and statistical methods. The matching scores and number of minutiae points are plotted against the respective testing variables for both capacitive and optical fingerprint sensor. The users are assumed to have healthy biometrics and they represent all age groups, demographics and both of the genders. The environmental factors like humidity, temperature and lighting should be simulated "normal operating conditions" as stated by the vendor and should be held as constant as possible throughout the testing.

Figure 5.1 and 5.2 shows the average match score (optical and capacitive sensor respectively) of eighty users for different sensor cleaning frequencies when compared against a genuine user's fingerprint captured with a clean sensor and normal conditions. The maximum frequency of sensor cleaning I have considered is hundred and I have taken average of every 5 times sensor is used. It can be inferred from the bar graph that sensor cleaning does not affect optical fingerprint sensor at all, atleast upto 100 touches. Also it can be deduced that the sensor cleaning has a substantial affect on capacitive sensor as compared to optical sensor. The plot shows a continuous degradation in performance of the sensor when number of touches increases. 


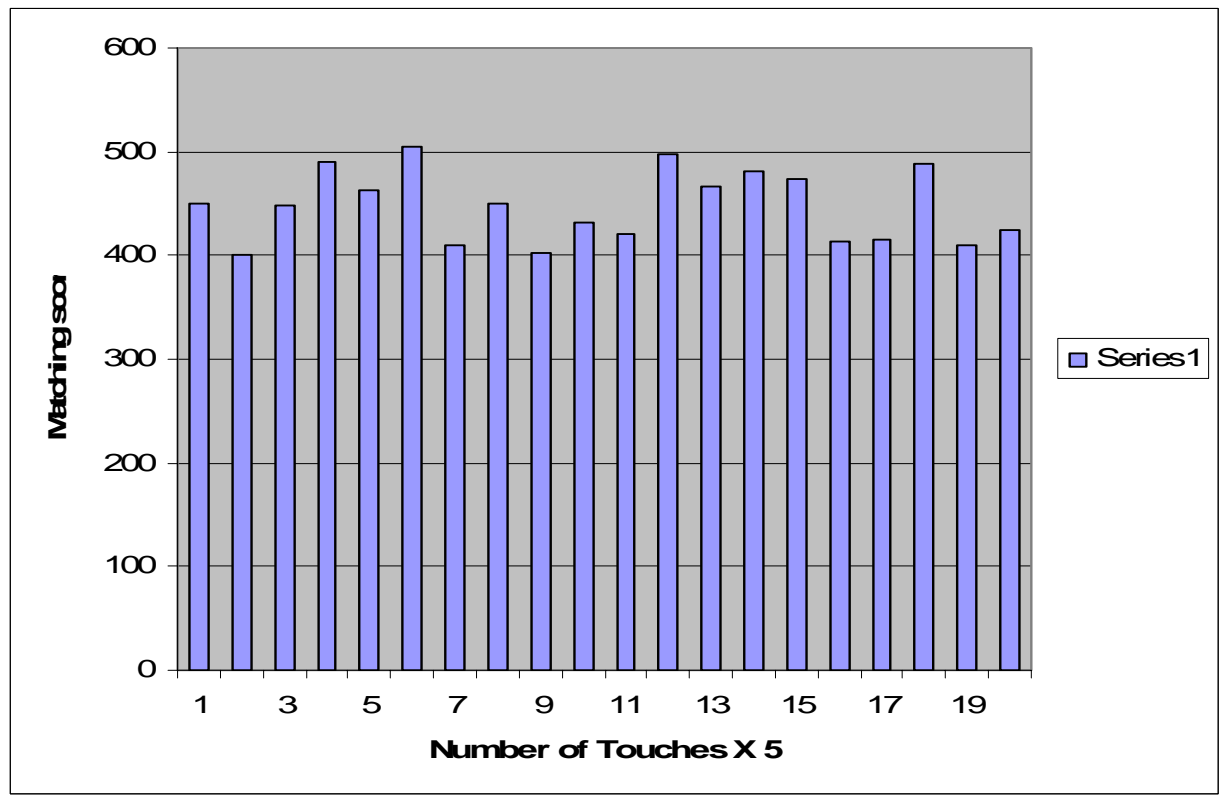

Figure 5.1 Average Genuine Match Score for Number of Sensor touches (Optical Sensor)

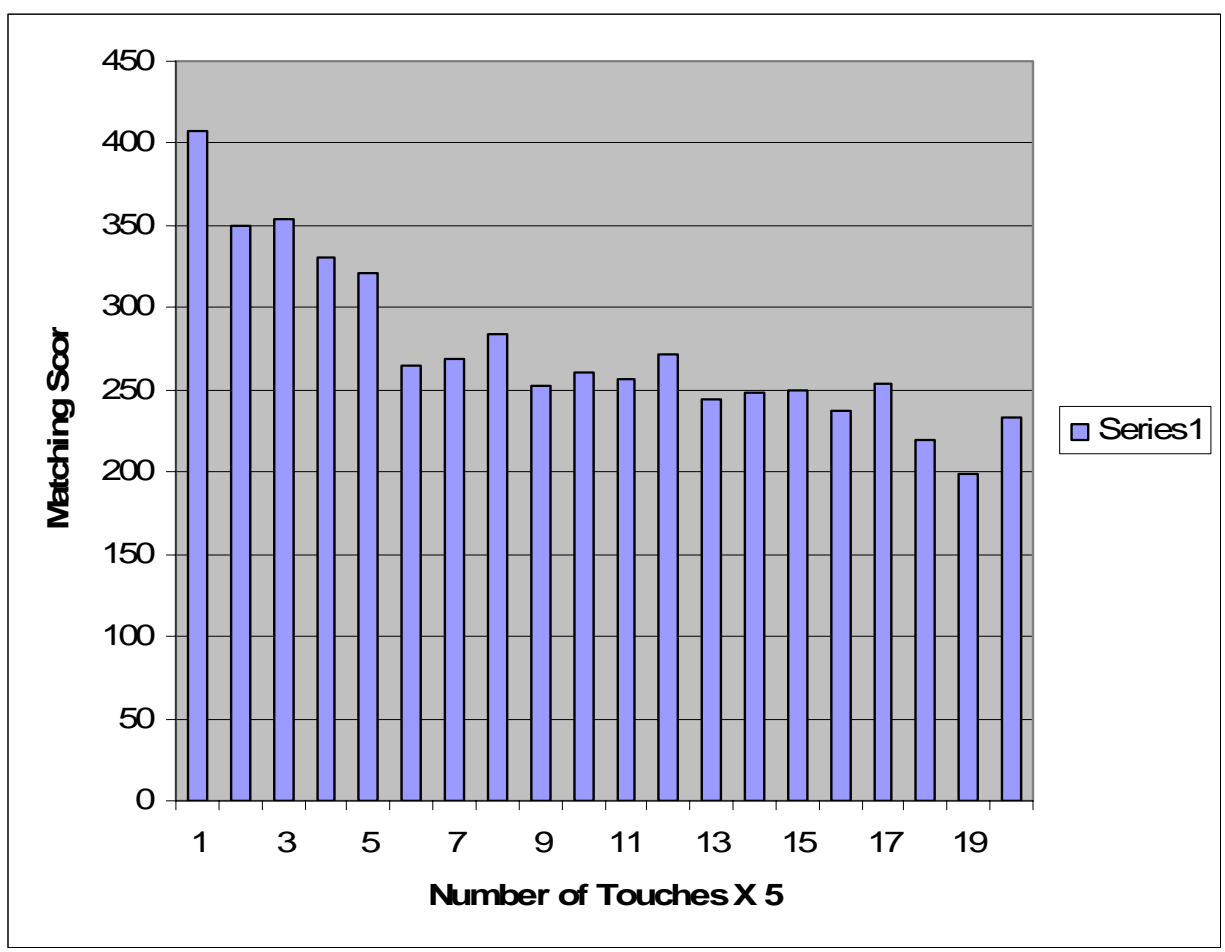

Figure 5.2 Average Genuine Match Score for Number of Sensor touches (Capacitive Sensor) 
Figure 5.3 and 5.4 shows the average number of minutiae points (optical and capacitive sensor respectively) of eighty users for different sensor cleaning frequencies when compared against a genuine user's fingerprint captured with a clean sensor and normal conditions. The maximum frequency of sensor cleaning I have considered is hundred and I have taken average of every 5 times sensor is used. It should be noted that the sensor cleaning has no affect on number of minutiae points for optical sensor but for capacitive sensor number of minutiae points decreases as number of touches without sensor cleaning increases.

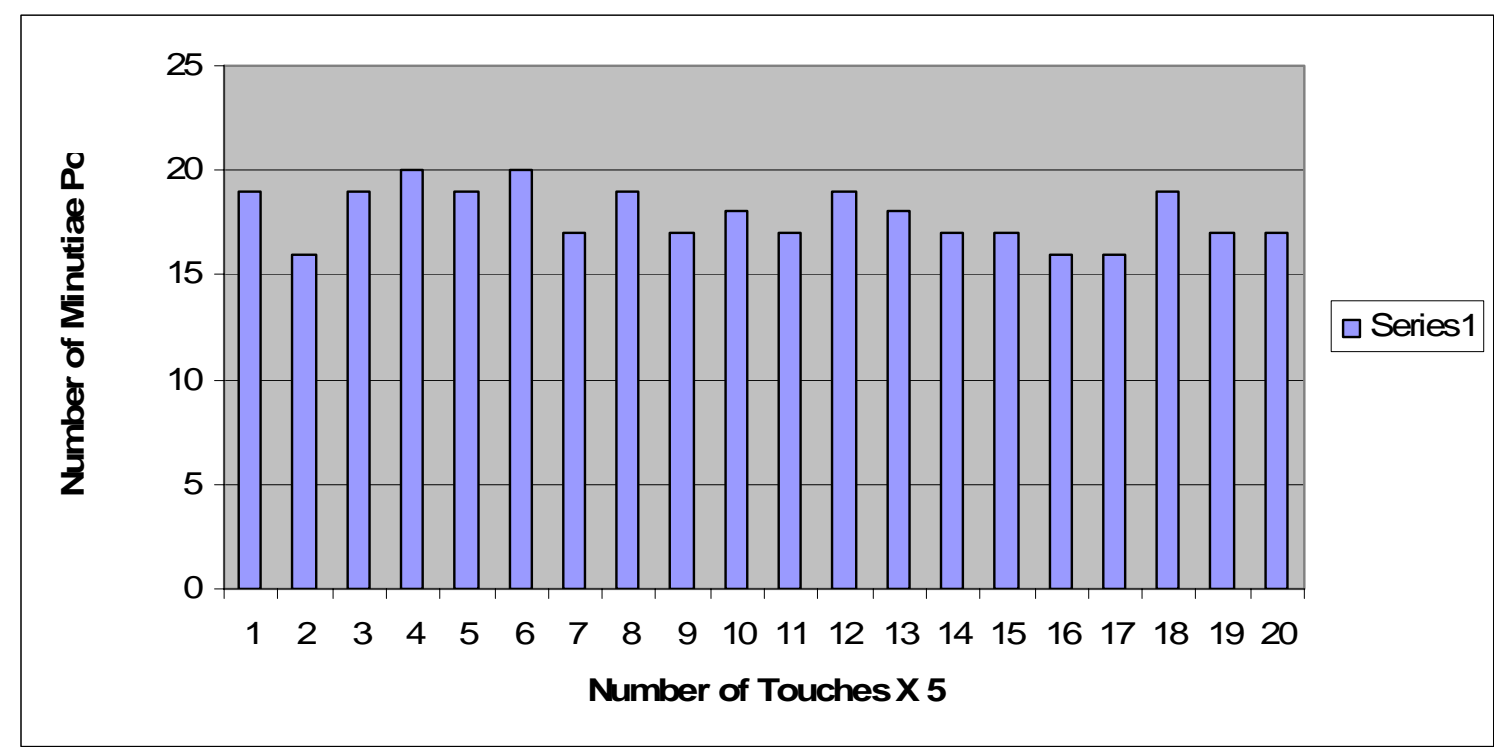

Figure 5.3 Average Number of Minutiae points for Number of Sensor touches

\section{(Optical Sensor)}




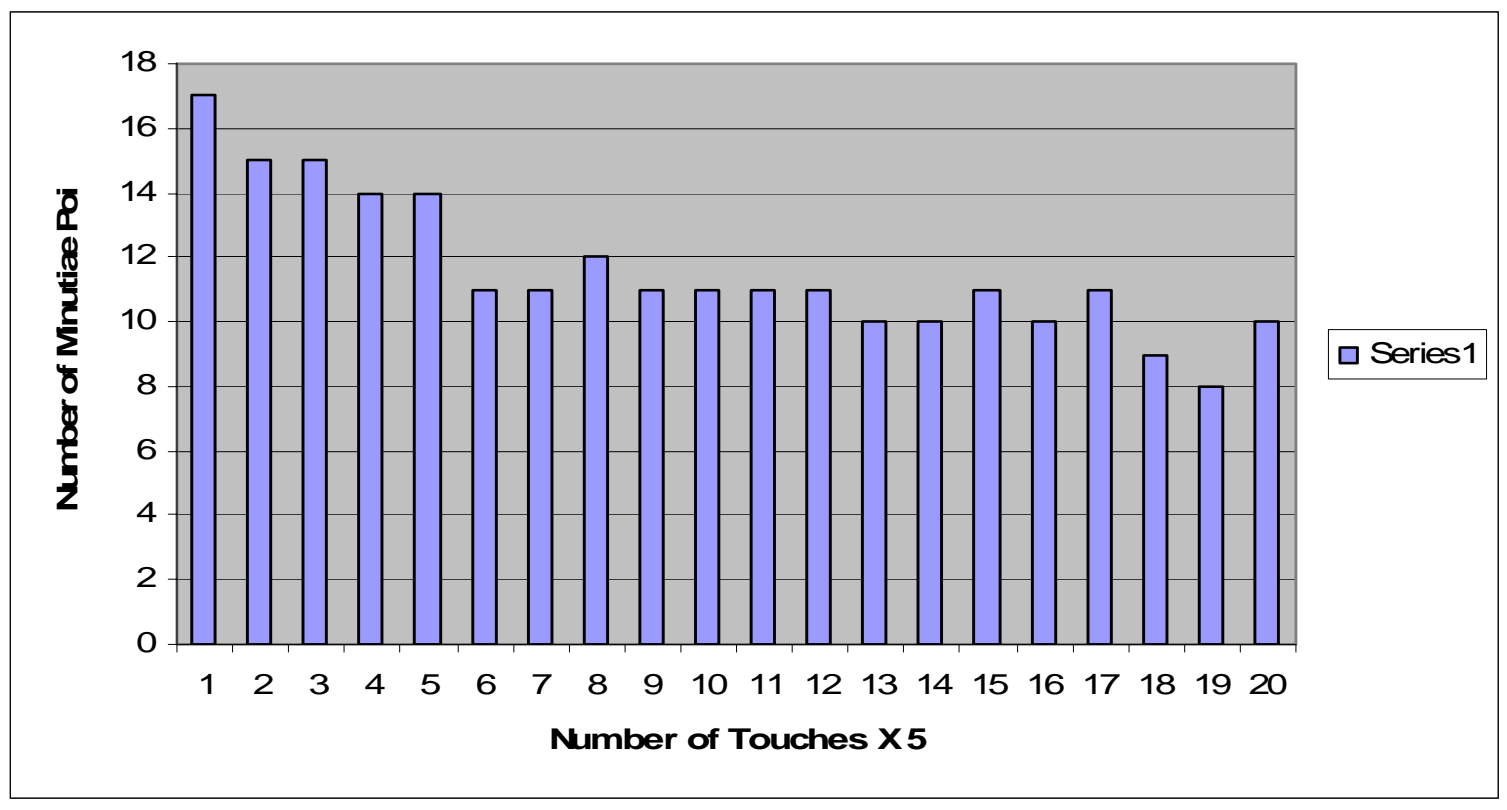

Figure 5.4 Average Number of Minutiae points for Number of Sensor touches (Optical Sensor)

Figure 5.5 and 5.6 shows the average match score (optical and capacitive sensor respectively) of eighty users for change in temperature when compared against a genuine user's fingerprint captured with a clean sensor and normal conditions. The normal operating temperature assumed is 71 degrees. It can be inferred from the bar graph that change in temperature does not have significant affect on both optical and capacitive fingerprint sensors. Though it can be seen that performance is comparatively better for temperature higher than the normal temperature. 


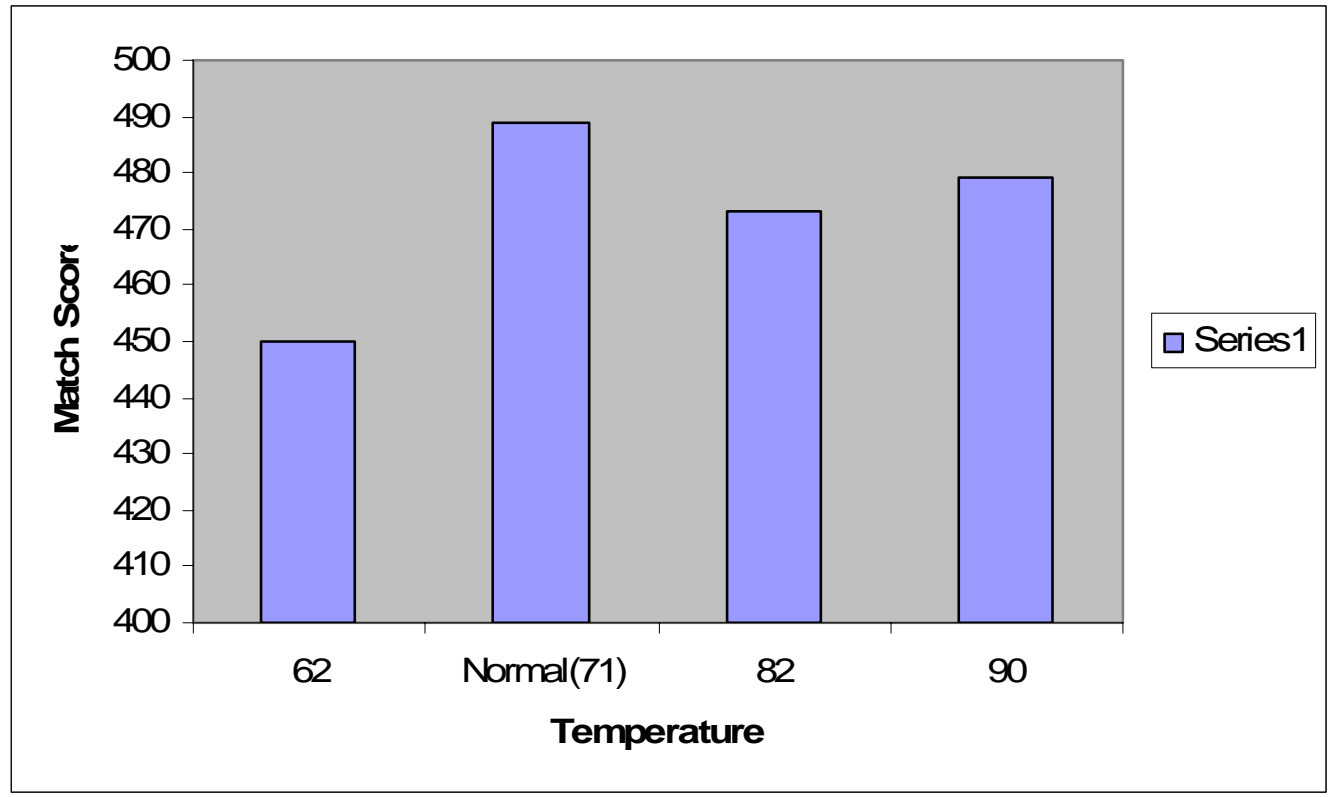

Figure 5.5 Average Genuine Match Score for Change in Temperature in

Faraheneit (Optical Sensor)

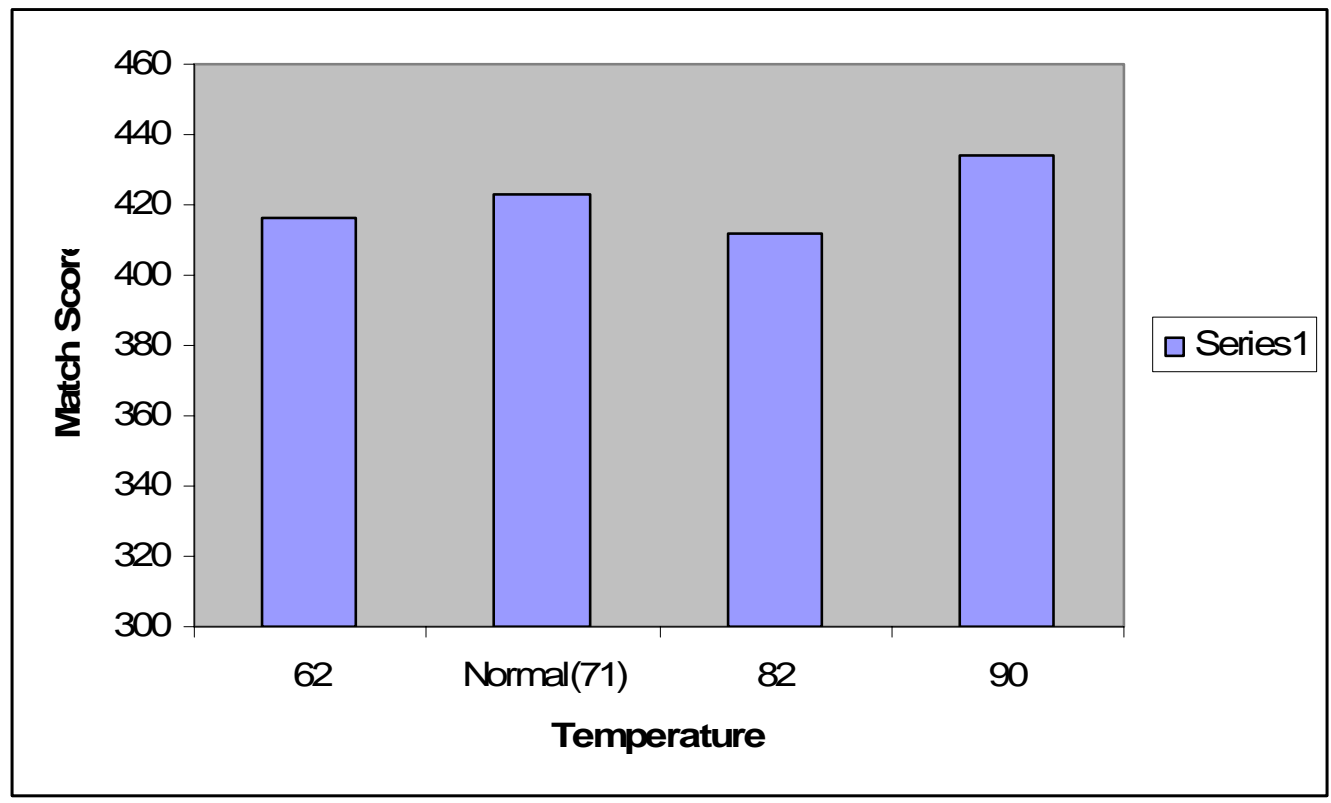

Figure 5.6 Average Genuine Match Score for Change in Temperature in

Faraheneit (Capacitive Sensor) 
Figure 5.7 and 5.8 shows the average number of minutiae points (optical and capacitive sensor respectively) of eighty users for different temperatures when compared against a genuine user's fingerprint captured with a clean sensor and normal conditions. The normal operating temperature assumed is 71 degrees. It should be noted from the bar graph that change in temperature does not have significance affect on both optical and capacitive fingerprint sensors. Though for capacitive sensor it can be seen that the performance is same at 90 degrees as compare to normal temperature.

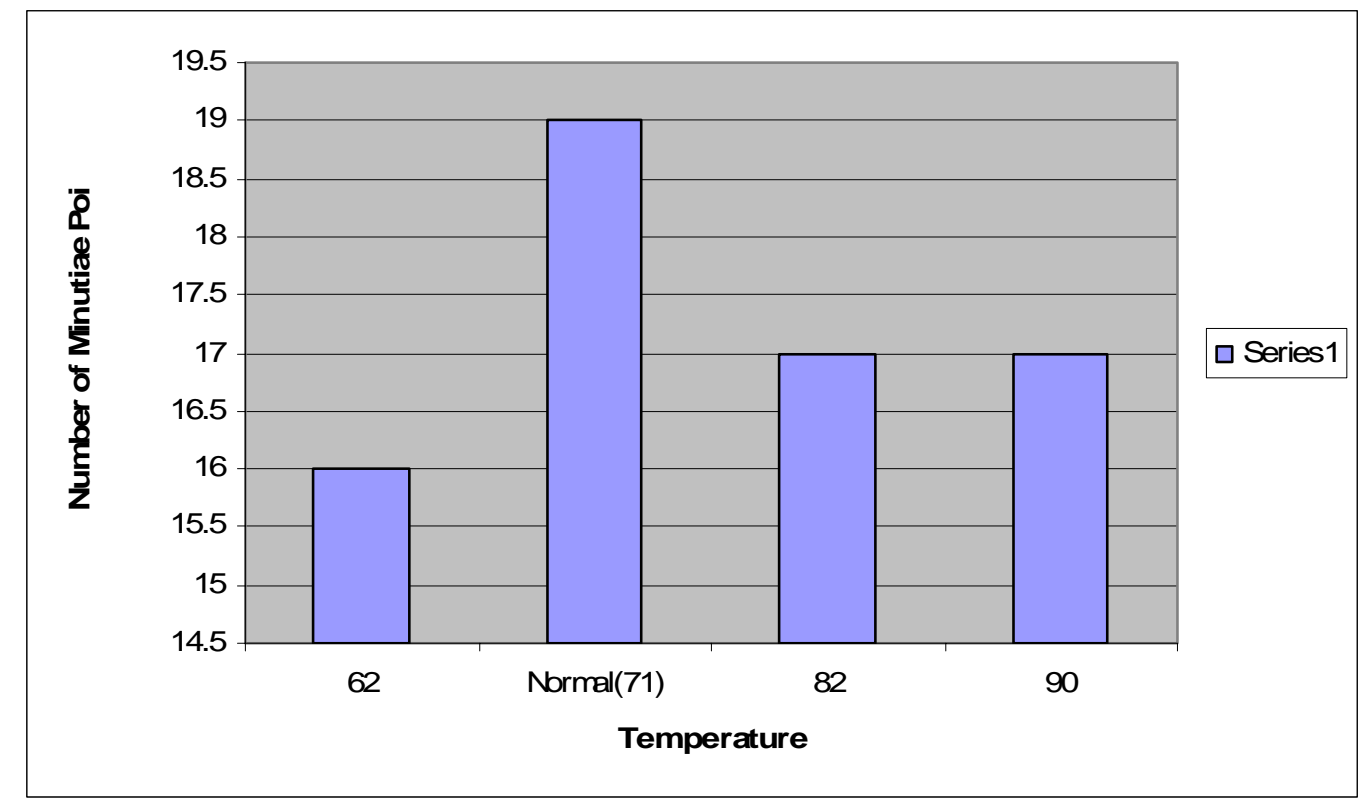

Figure 5.7 Average Number of Minutiae points for Temperature Change (Optical

Sensor) 


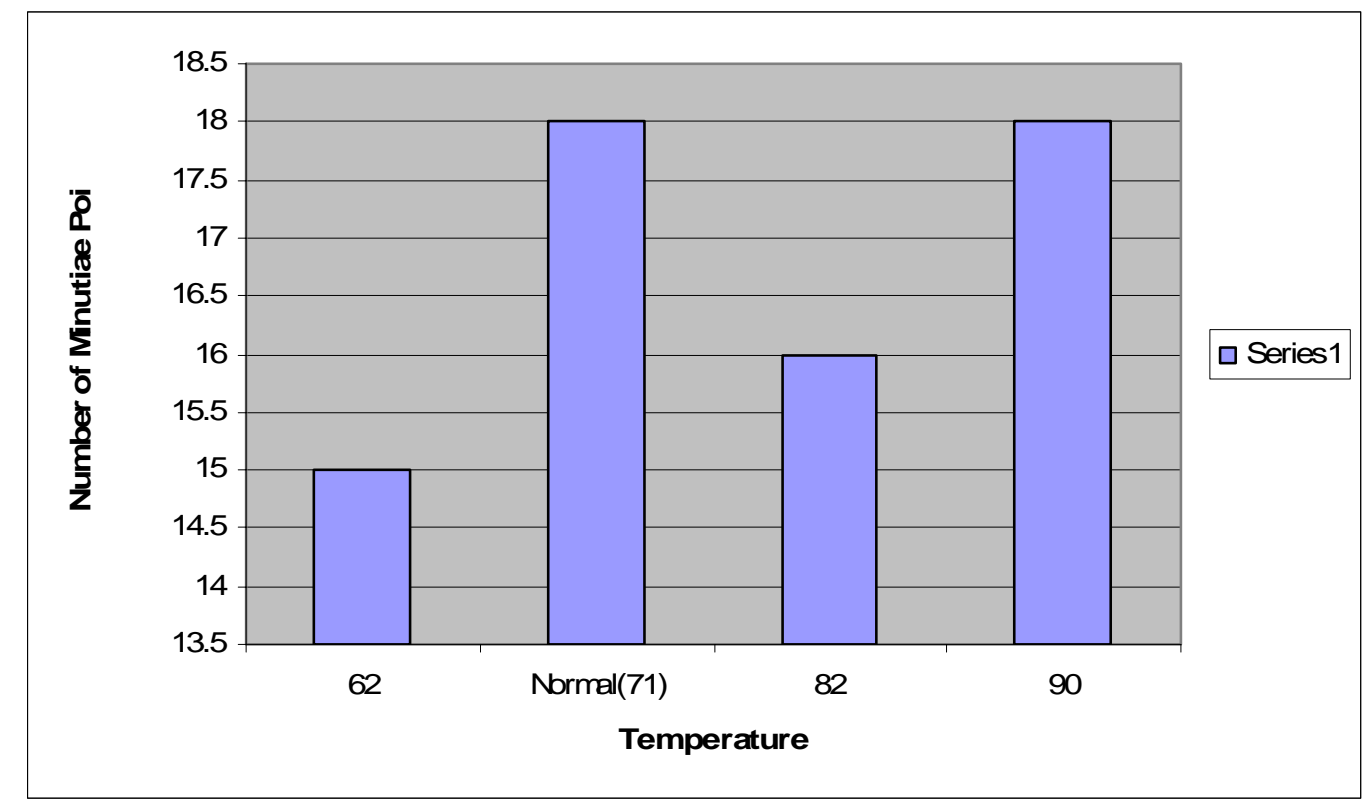

Figure 5.8 Average Number of Minutiae points for Temperature Change

(Capacitive Sensor)

Figure 5.9 and 5.10 shows the average genuine match score (optical and capacitive sensor respectively) of eighty users for change in device placement (Pan $+/-20$, Pan $+/-10$, Tilt $+/-20$, Tilt $+/-10$ ) when compared against a genuine user's fingerprint captured with a clean sensor and pan and tilt angle of zero degrees. It can be inferred from the bar graph that the performance of the sensor is better for pan and tilt angles of $-20 /-10$ as compared to pan and tilt angles of $+20 /+10$ degrees. This shows that the user is more comfortable with negative angles as compared with positive angles. 


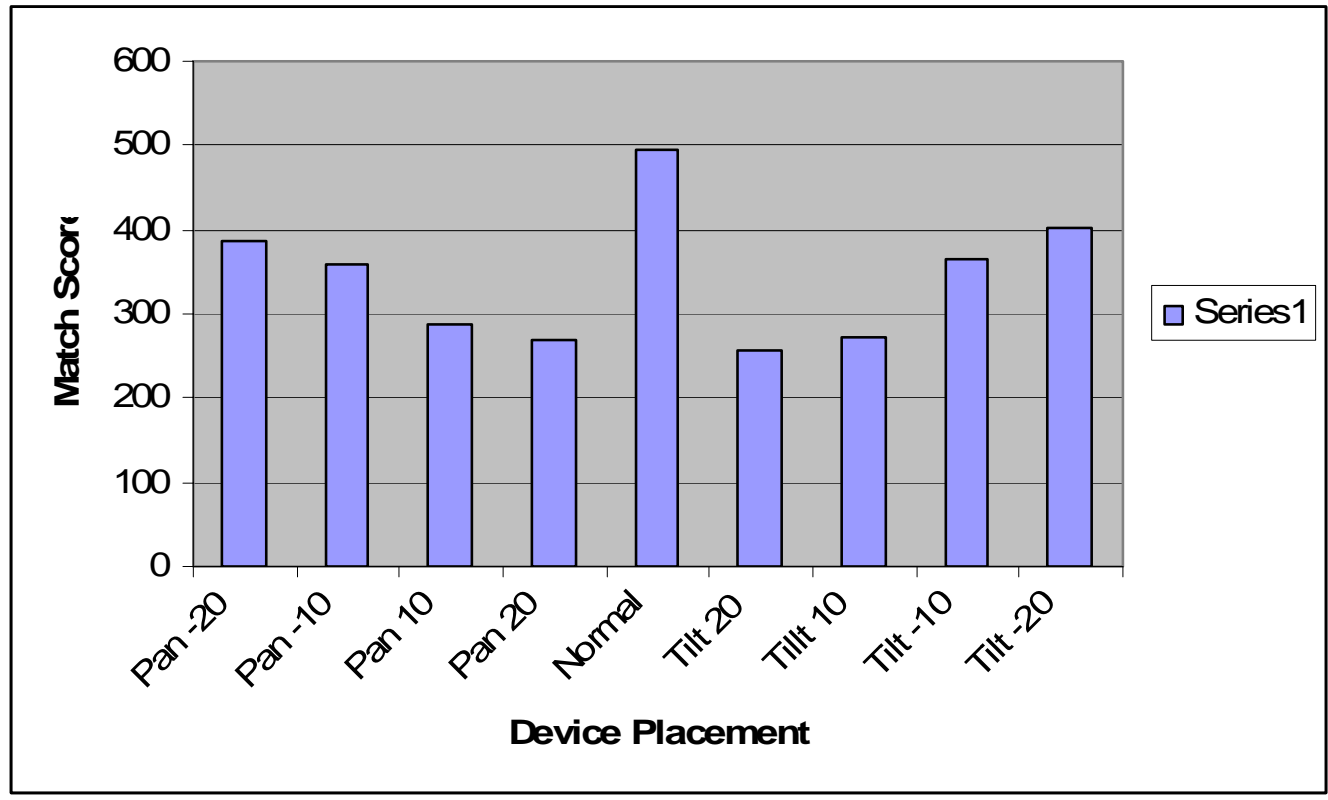

Figure 5.9 Average Genuine Match Score for Device Placement (Optical

Sensor)

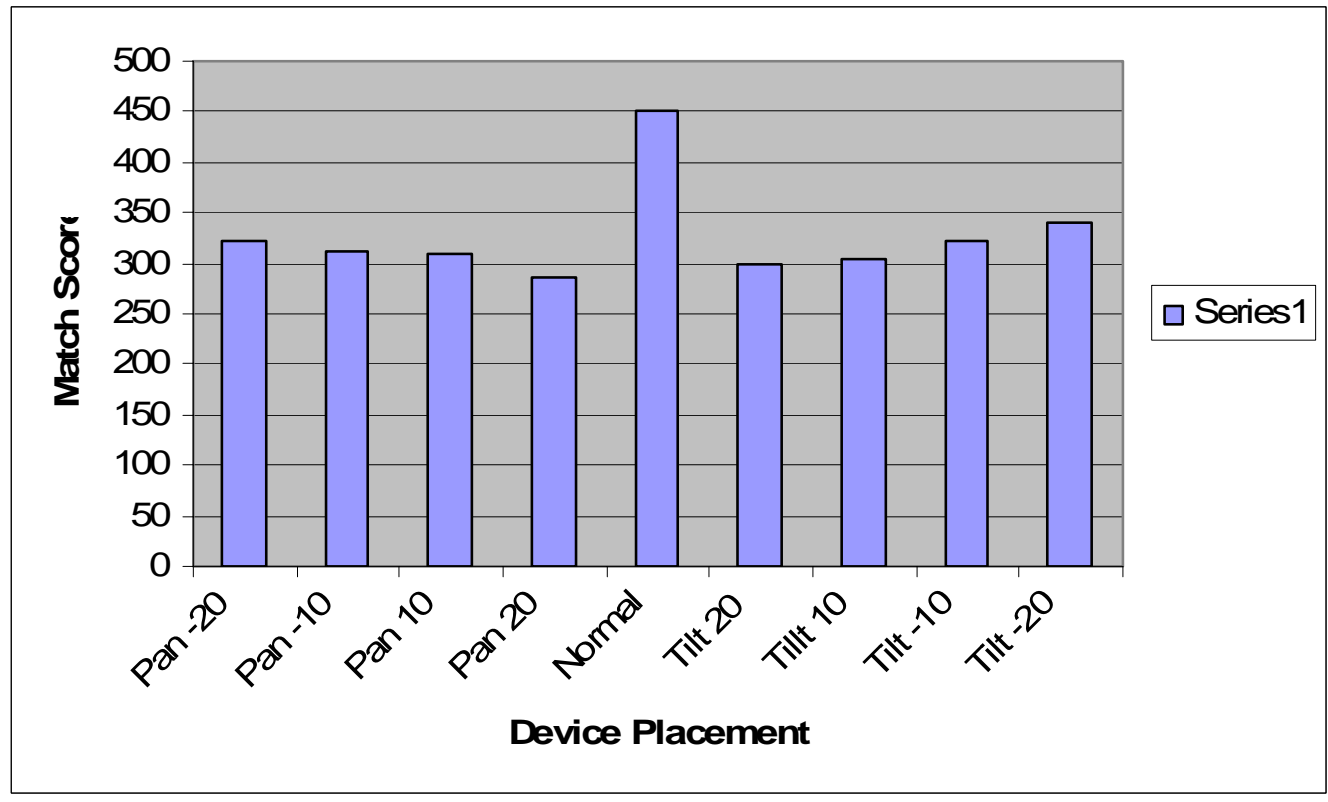

Figure 5.10 Average Genuine Match Score for Device Placement (Capacitive Sensor) 
Figure 5.11 and 5.12 shows the average number of minutiae points (optical and capacitive sensor respectively) of eighty users for different change in device placement (Pan $+/-20$, Pan $+/-10$, Tilt $+/-20$, Tilt $+/-10)$ when compared against a genuine user's fingerprint captured with a clean sensor and pan and tilt degrees of zero. Again the numbers of minutiae points are more for negative angles as compared to positive angles.

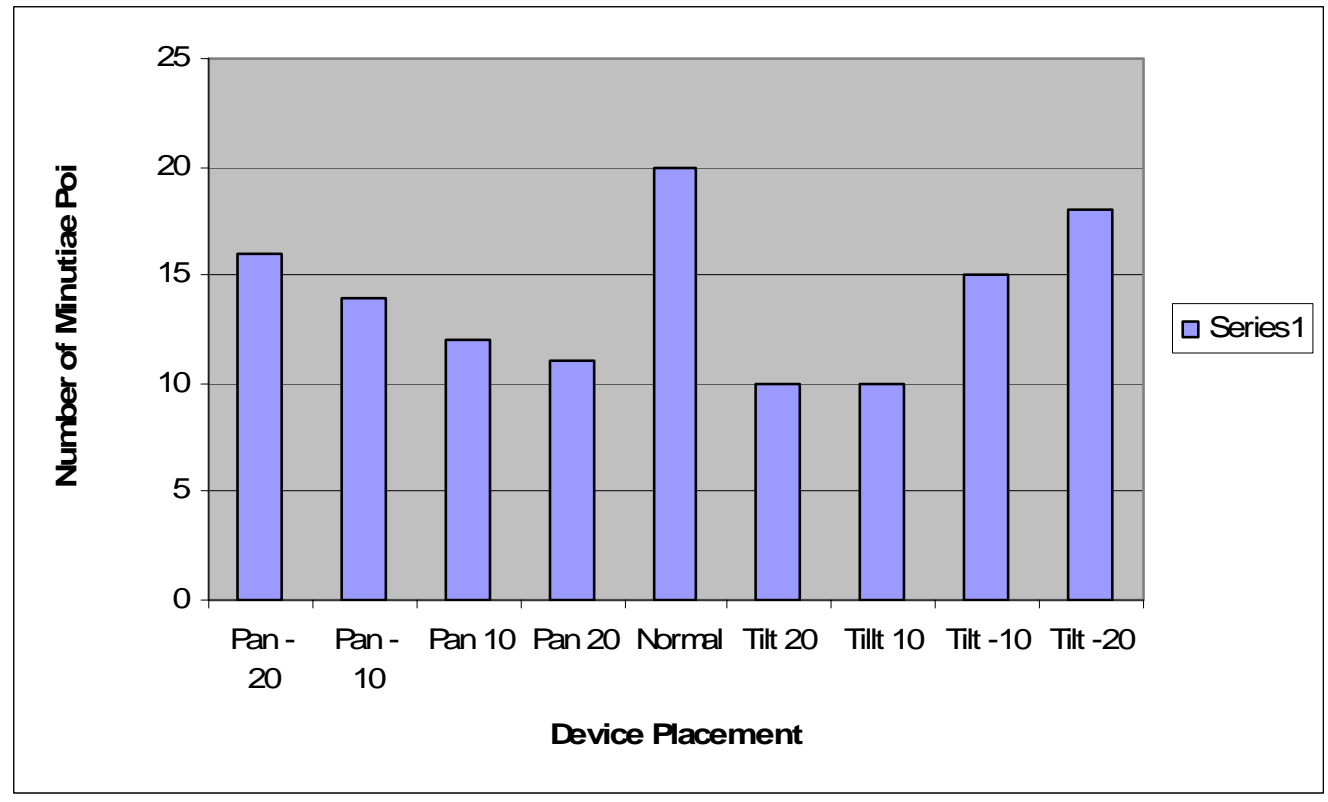

Figure 5.11 Average Number of Minutiae points for Device Placement (Optical

Sensor) 


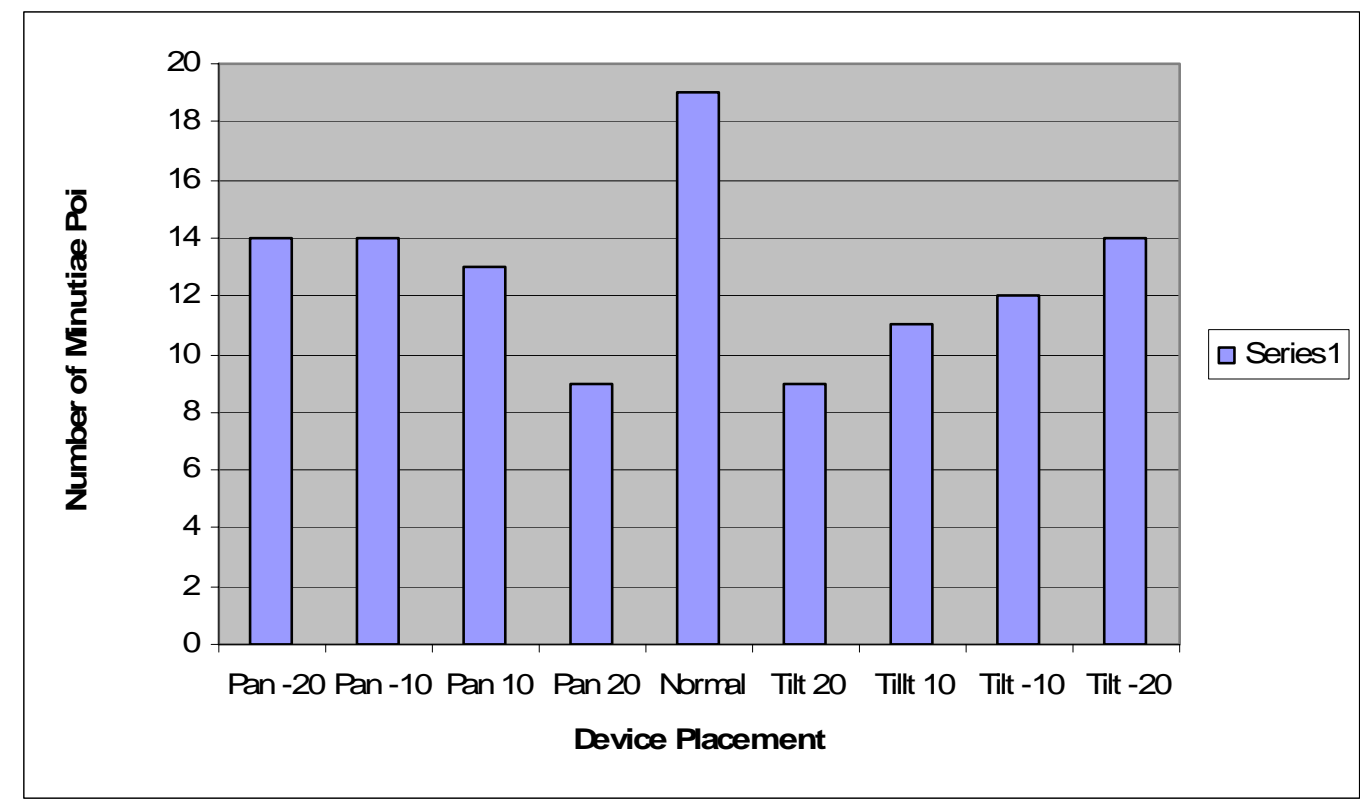

Figure 5.12 Average Number of Minutiae points for Device Placement (Capacitive Sensor)

Figure 5.13 and 5.14 shows the average genuine match score (optical and capacitive sensor respectively) of eighty users for various angles of rotation when compared against a genuine user's fingerprint captured with a clean sensor and user's fingerprint at 90 degrees angle of rotation. The angles considered are 45, 60, 85, 95, 120 and 135 degrees. It should be noted that performance continuously decreases as the angle increases or decreases from the normal position of 90 degrees. Moreover performance is better for lower angles as compared to the higher angles for optical sensor and vice-versa for capacitive sensor. 


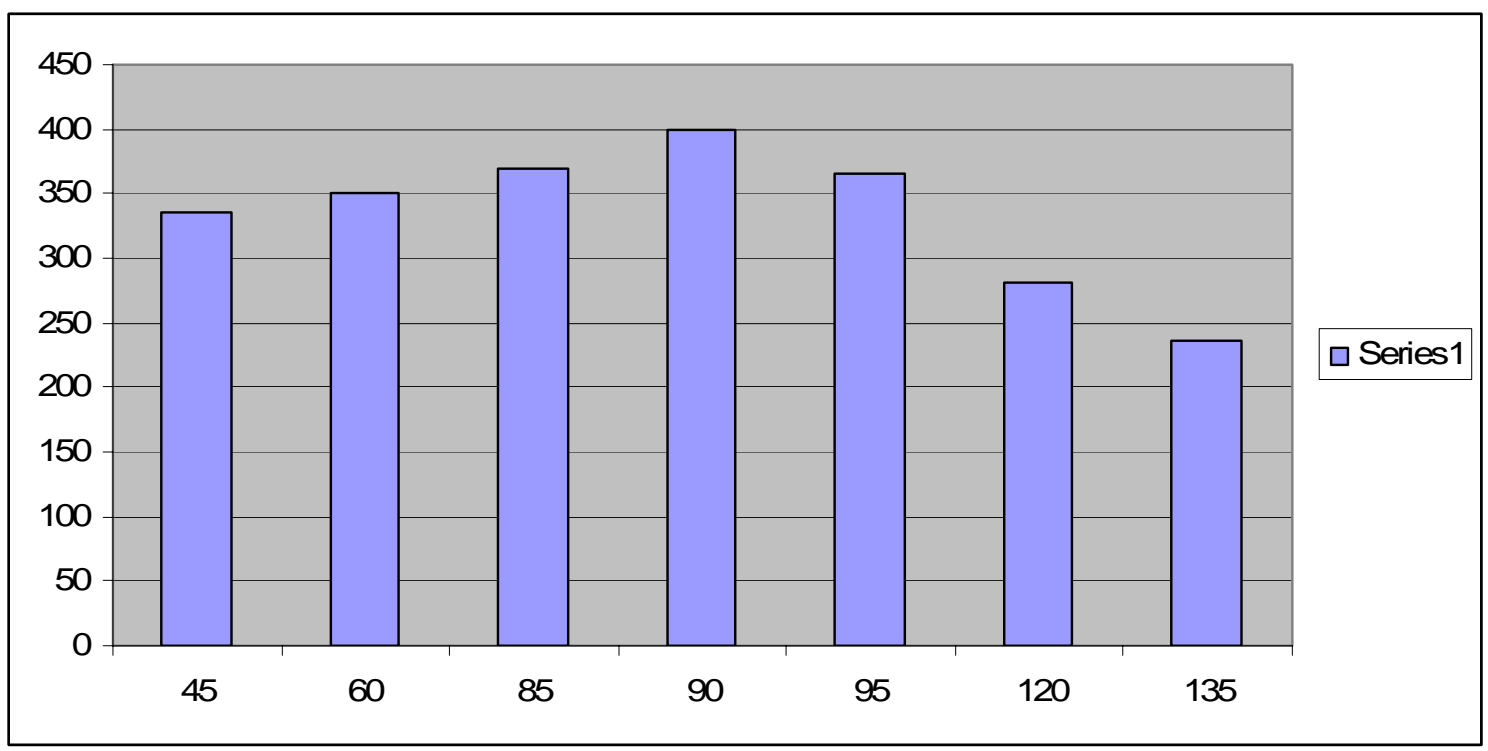

Figure 5.13 Average Genuine Match Score for Biometric Presentation in degrees

(Optical Sensor)

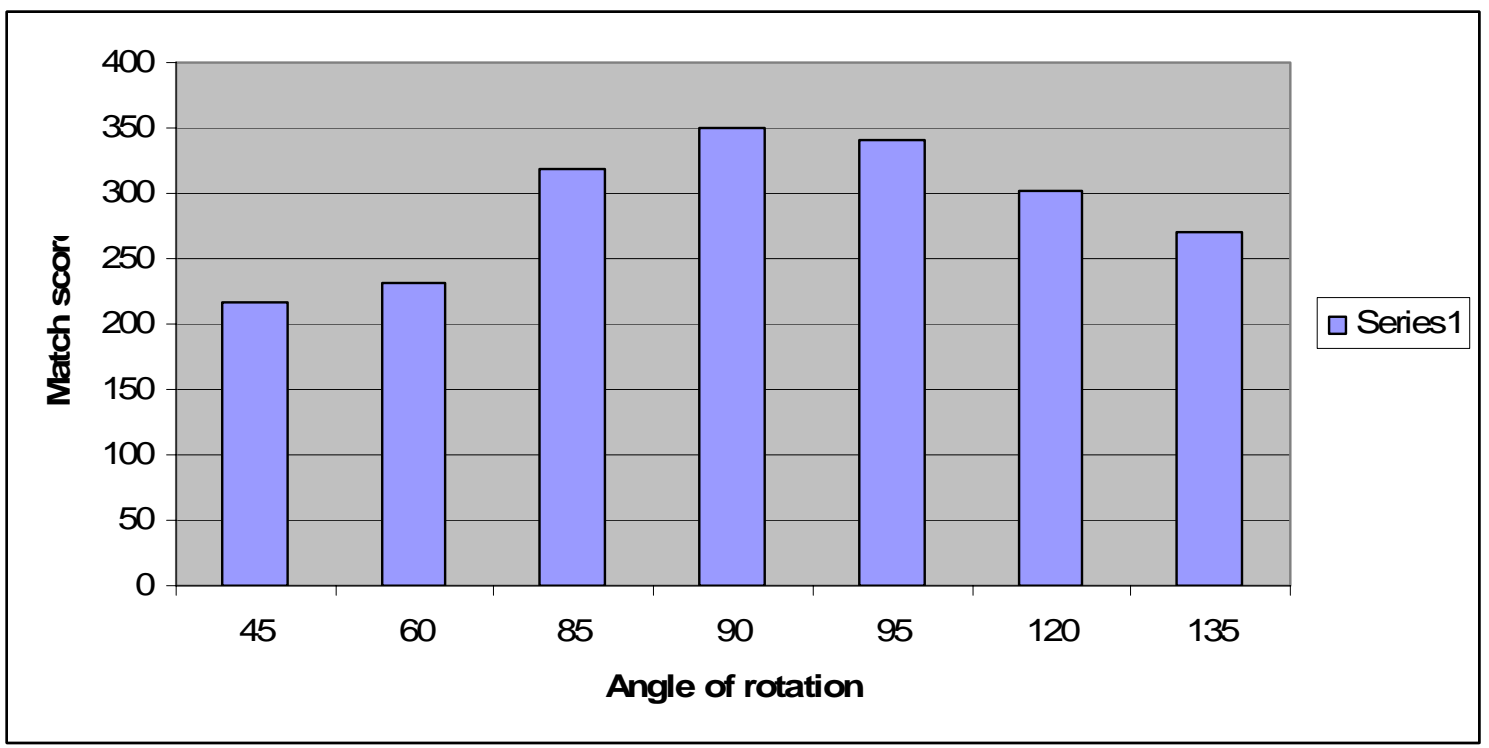

Figure 5.14 Average Genuine Match Score for Biometric Presentation in degrees (Capacitive Sensor) 
Figure 5.15 and 5.16 shows the average number of minutiae points (optical and capacitive sensor respectively) of eighty users for various angles of rotation when compared against a genuine user's fingerprint captured with a clean sensor and user's fingerprint at 90 degrees angle of rotation. Again the numbers of minutiae points are more for lower angles for optical sensor and less for capacitive sensor.

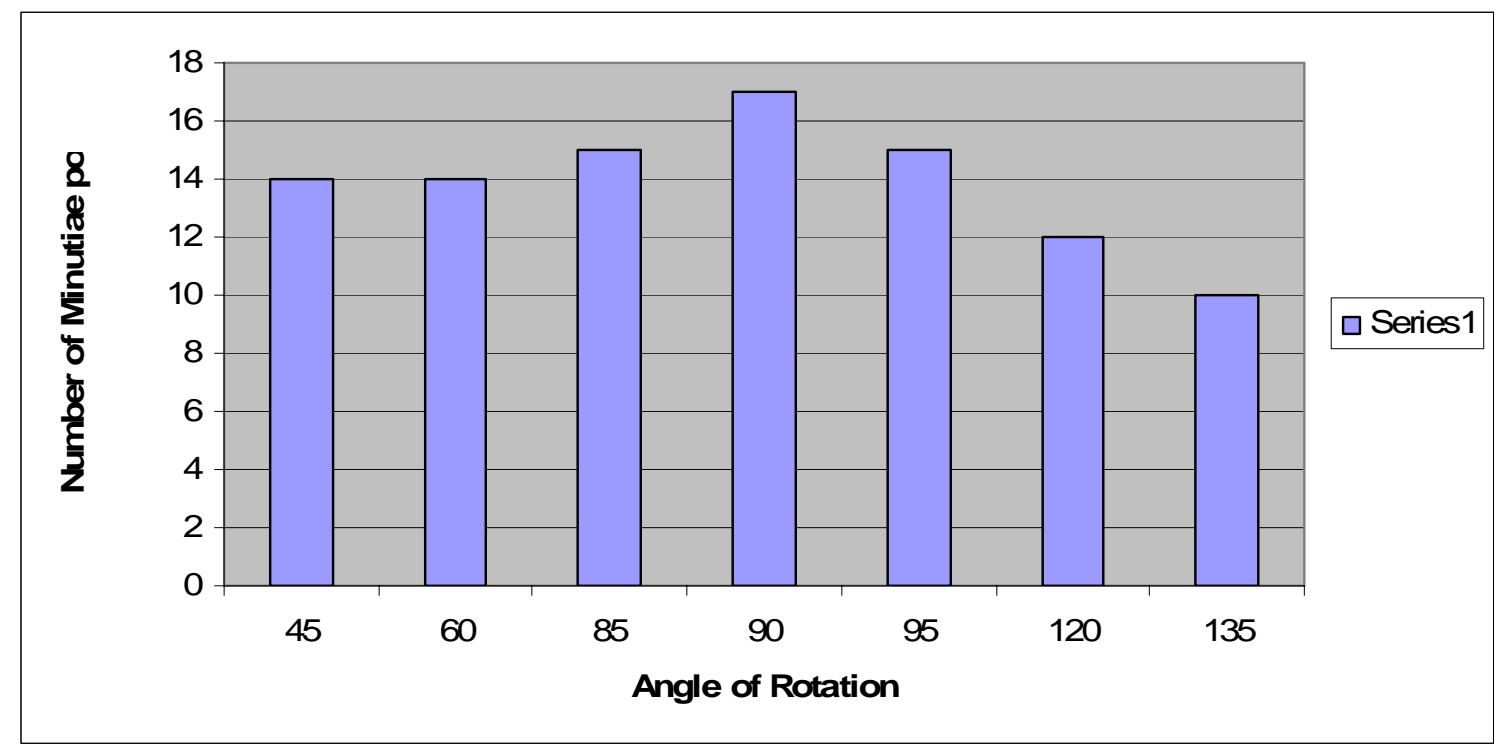

Figure 5.15 Average Number of Minutiae points for Angle of Rotation (Optical Sensor) 


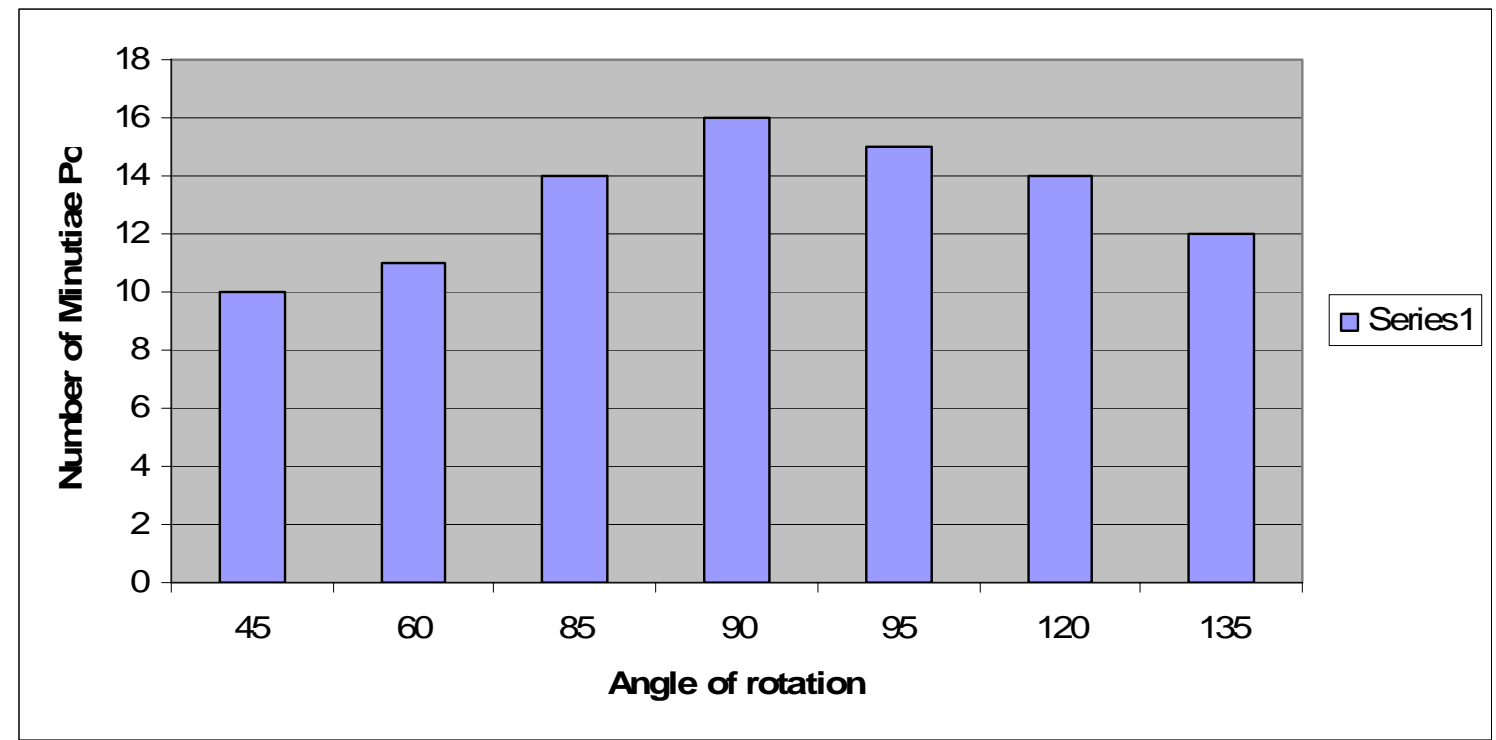

Figure 5.16 Average Number of Minutiae points for Angle of Rotation (Capacitive

Sensor) 


\section{CHAPTER 6 ANALYSIS AND FUTURE WORK}

\subsection{Comparison between optical an capacitive sensor}

As can be interpreted from the results shown in the last chapter all the variables considered does affect the fingerprint sensor performance though in different ways depending on the type of sensor used.

Optical fingerprint sensor proved to be robust against sensor surface cleaning. I performed the experiment for 100 sensor touches and the performance of the sensor was not affected by the variable (See Fig 5.1). As far as capacitive sensor is concerned it is highly prone to the sensor surface cleaning. As can be seen from Fig 5.2 the performance drops continuously as the number of touches increases proving the vulnerability of sensor against the variable. The reason may be that in real world environments sensors become soiled due to repeated contact with user's fingers and external contamination which leads to change in distances of ridges and valleys from the micro-capacitor plate as compared to the clean sensor.

Temperature is an issue with optical sensor as the performance fluctuates with change in temperature which is unlike capacitive sensor. The performance of capacitive sensor does not change much with change in temperature. Another important observation is that for both the sensors performance is better for above normal temperature as compared with below normal temperature.

The device placement considers the effects of placing your biometric device at different positions. The variable affects both the sensor in pretty much the same way. An interesting phenomenon here observed is that the performance of the sensor is better for pan and tilt angles of $-20 /-10$ as compared to pan and tilt angles of $+20 /+10$ degrees. This 
shows that the user is more comfortable with device placed at negative angles as compared with positive angles. This is more of a human factor related as it shows the comfortableness of user with negative pan and tilt angles as compared to positive angles. For various angles of rotation of finger the performance continuously decreases as the angle increases or decreases from the normal position of 90 degrees. Moreover performance is better for lower angles as compared to the higher angles for optical sensor and vice-versa for capacitive sensor. This makes sense as far as optical sensor is concerned because the arrangement of light source and prism might be inclined towards lower angles.

The above mentioned affects on fingerprint sensors can also be due to the use of different image capture software. For optical sensor I have used Secugen software and for capacitive I have used Authentec software.

\subsection{Curve fitting and prediction}

Another main objective of this thesis is to fit a curve on the performance characteristics so that the performance can be predicted beforehand if the value of the variable is known. For example if we know that this is the fiftieth touch to the sensor without cleaning we could lower the threshold accordingly and hence we can make the matching algorithm to learn automatically. Because of lowering of threshold the user who would have been rejected is now accepted and hence the applications where FRR is critical as compared to FAR this technique will be very useful.

- Sensor cleaning frequency for capacitive sensor: As mentioned above sensor cleaning does not affect optical sensor and hence I have done curve fitting on capacitive sensor only. I have data for 100 touches only and that's why I took 0 
and 100 as my boundaries for curve fitting. I have used non linear regression using GraphPad PRISM software for WINDOWS to fit a curve on my data. The second order polynomial used is of the form

$$
\mathrm{Y}=\mathrm{A}+\mathrm{B} * \mathrm{X}+\mathrm{C}^{*} \mathrm{X}^{\wedge} 2
$$

The best-fit values for A, B and C are 398.5, -19.25 and 0.5497 respectively (Fig 6.1) that gives $\mathrm{R}^{2}$ value of 0.8814 and Sy.x (Relative sum of squares) value of 18.82. So now for sensor cleaning variable the above mentioned equation is used to predict the match score values if number of touches are known beforehand. This value can be used to adjust the threshold accordingly.

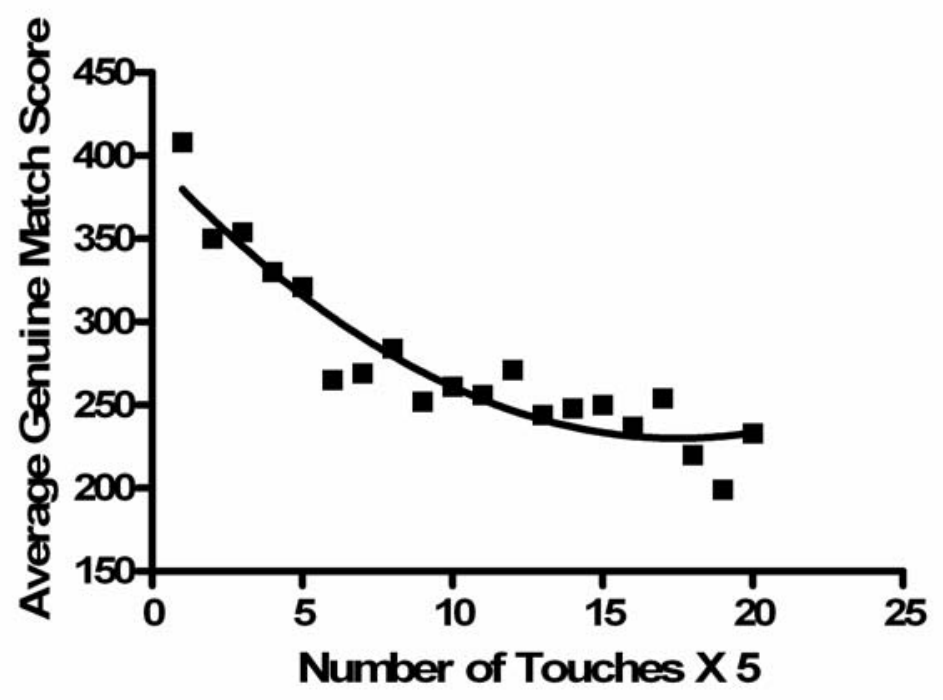

Figure 6.1 Curve fitting using non linear regression for change in sensor cleaning frequency (capacitive sensor)

- Angle of Rotation: I have data for seven angles from 45 degrees to 135 degrees so these acts as our boundaries for curve fitting. I have used non linear regression to fit a curve on my data. The second order polynomial used is of the form 


$$
\mathrm{Y}=\mathrm{A}+\mathrm{B}^{*} \mathrm{X}+\mathrm{C}^{*} \mathrm{X}^{\wedge} 2
$$

The best-fit values for A, B and C are $-121.5,2.238$ and -0.0467 respectively (Fig 6.2) that gives $R^{2}$ value of 0.8787 and Sy.x (Relative sum of squares) value of 22.23 for capacitive sensor. For optical sensor the best-fit values for A, B and C are $94.89,7.292$ and -0.04668 respectively (Fig 6.3) that gives $\mathrm{R}^{2}$ value of 0.9314 and Sy.x (Relative sum of squares) value of 18.12. So now for angle of rotation variable the above mentioned equation is used to predict the match score values if angle of rotation is known. This value can be used to adjust the threshold accordingly.

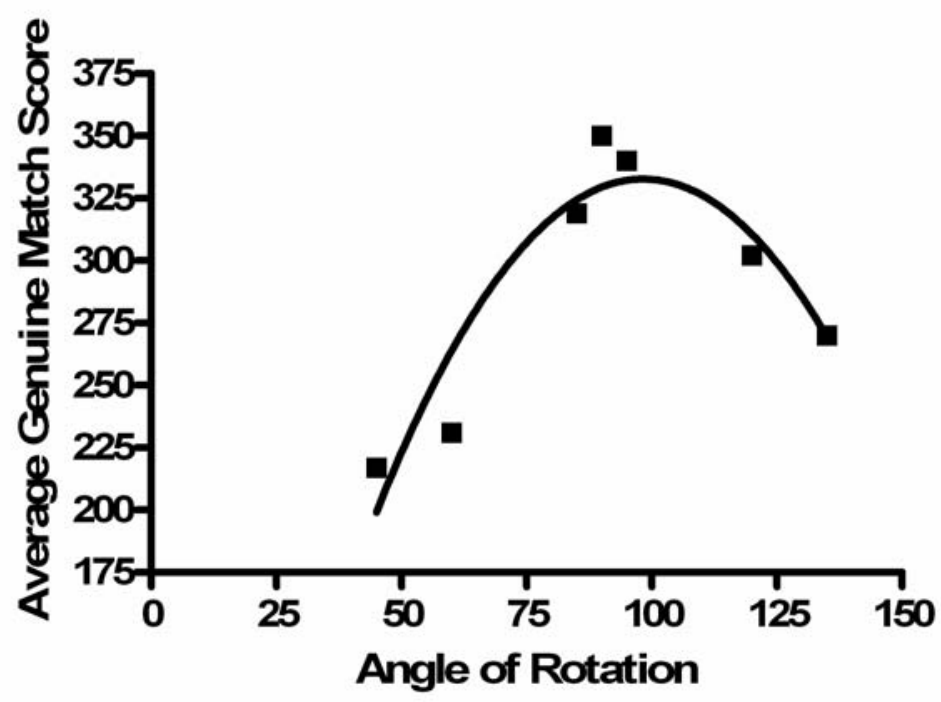

Figure 6.2 Curve fitting using non linear regression for change in angle of rotation (capacitive sensor) 


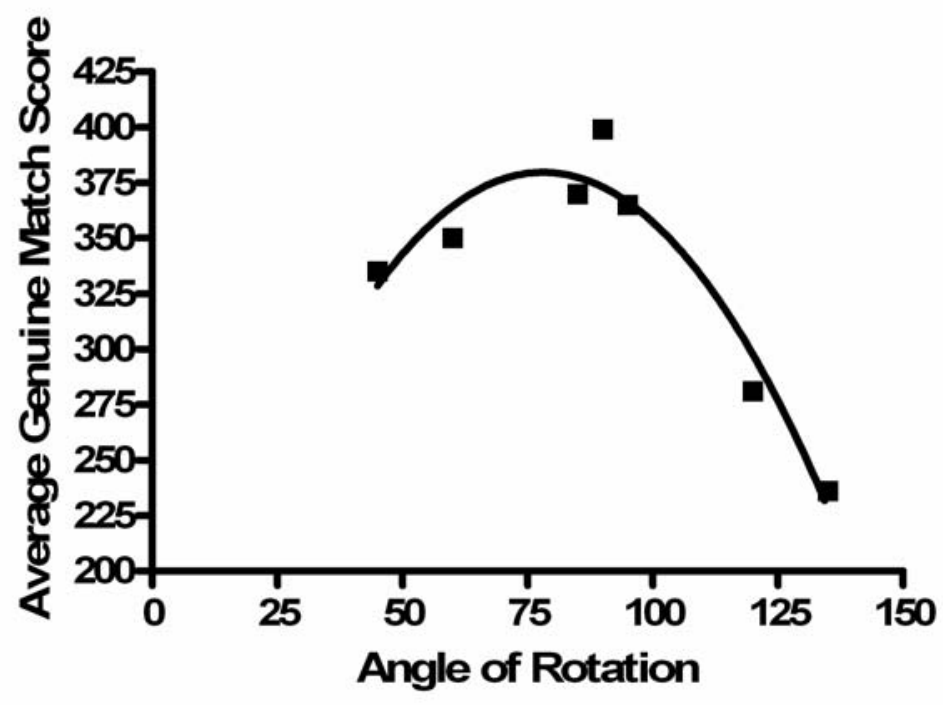

Figure 6.3 Curve fitting using non linear regression for change in angle of rotation (optical sensor)

For other two variables considered that is temperature and device placement the data was not sufficient enough to perform curve fitting or modeling.

\subsection{Future work}

The testing and evaluation of biometric devices is still in its infancy and there are many problems that can be addressed in future work. The main problem is the number of users that ensures the validity of the results. I have used 80 users but testing the above protocols with more test subjects is necessary to get a more accurate representation of imposter and genuine distributions, and will lead to better modeling of these variables. For modeling of temperature and device placement the data range of these variables should be higher with low least count.

Also the correlation between different factors can be modeled from the individual models of the factors. This will help in predicting performance beforehand if the values of all the variables are known. This will save data collection time as we don't have to collect data 
for all the combination of variables, instead the data is collected for individual factors and later correlation between multiple factor is calculated and modeled.

I tried to compare the optical and capacitive sensor using human and environmental factors as the criterion but there should be some other methodologies developed to compare these sensors.

Another important work that can be done is use of environmentally controlled chambers so that all the variables considered can be precisely controlled.

The curve fitting results can be actually applied or augmented with the fingerprint recognition algorithm so that the algorithm can be tuned according to the variable and hence FRR can be reduced.

The protocols repeatability and generality are other issues that need more work. 


\section{References}

[1] Ashbourn, J. (2000). Biometrics: Advanced Identity Verification. Springer, London.

[2] Bolle, R. M., Pankanti, S., \& Ratha, N. K. (2000). Evalution Techniques for

Biometrics-Based Authentication systems (FRR). Yorktown Heights, NY: IBM.

[3] Bolle, R.M., Ratha, N., K., and Pankanti, S. Confidence interval measurement in performance analysis of biometric systems using the bootstrap. Proceedings of Workshop on Empirical Evaluation Methods in Computer Vision, Hawaii, December 2001.

[4] Doddington, G. R., Pryzbocki, M. A., Martin, A. F., \& Reynolds, D. A. (2000). The NIST Speaker Recognition Evaluation: Overview Methodology, Systems, Results, Perspective (31). Speech Communications.

[5] Efron, B. and Tibshirani, R.J. An introduction to the bootstrap, Chapman and Hall, 1997.

[6] Fingerprint Verification Competition. Retrieved on 04/12/2005 from: http://bias.csr.unibo.it/fvc2004/default.asp 
[7] Fingerprint Vendor Technology Evaluation (FpVTE). Retrieved on 04/12/2005from:3 http://fpvte.nist.gov

[8] Holmes J. P., Wright L. J., Maxwell R. L., A Performance Evaluation of Biometric Identification Devices

[9] INCITS 1602-D Biometric Performance Testing and Reporting Part 3: Scenario Testing and Reporting Draft 3.0

[10] Increasing Accuracy in Multimodal Biometric Systems GIAC Security Essentials Certification (GSEC) Practical Assignment Version 1.4c Option

[11] Jain, A. K., \& Ross, A. (September 2002). Learning User-specific Parameters in a Multibiometric System, Proc. of IEEE International Conference on Image Processing (ICIP), (Rochester, NY), pp. 57-60.

[12] Jain, A. K., Ross, A., \& Prabhakar, S. (Eds.) (2004). An Introduction to Biometric Recognition. IEEE Transactions on Circuits and Systems for Video Technology, $14(4)$.

[13] Jain, A. K., L. Hong, and R. Bolle, “On-line fingerprint verification,” IEEE Transactions on Pattern Recognition and Machine Intelligence 19(4), pp. $302-$ 314, 1997.

[14] Jain A. K., R. Bolle and S. Pankanti (Eds.), BIOMETRICS: Personal Identification in Networked society, Kluwer Academic Publishers, 1999.

[15] Jain A. K. , S. Pankanti, S. Prabhakar, L. Hong, A. Ross, and J. L. Wayman, "Biometrics: A Grand Challenge", Proc. International Conference on Pattern Recognition (ICPR), (Cambridge, UK), Vol. 2, pp. 935-942, August 2004. 
[17] Kim, H. (2003). Evaluation of Fingerprint Readers: Environmental factors, Human Factors, \& Liveness Detecting Capability. Retrieved April 2, 2005 from: http://www.biometrics.org/bc2004/CD/PDF_PROCEEDINGS/Microsoft\%20Pow erPoint 20-\%20Presentation\%20of\%20HaleKim\%20-\%20v2.1.ppt\%20[.pdf

[18] Lawrence, O. (1999). Fingerprint Verification. In Jain, A., Bolle, R., \& Pankanti, S. (Eds.), Biometrics: Personal Identification in Networked Society (p. 43). Boston: Kluwer.

[19] Maltoni, D., Dario, M., Jain, A., \& Prabhakar, S. (2003). Handbook of Fingerprint Recognition. London: Springer.

[20] Mansfield, A. J., \& Wayman, J. L. (August 2002). Best Practices in Testing and Reporting Performances of Biometric Devices, Version 2.01 (NPL Report CMSC 14/02). UK: National Physical Laboratory.

[21] Mansfield, A.J., Kelly, G.P., Chandler, D.J., and Kane, J. Biometric product testing final report. Report for CESG and Biometrics Working Group, March 2001.http://www.cesg.gov.uk/technology/biometrics/media/Biometric\%20Test\%2 0Report\%20pt1.pdf

[22] Phillips P. J., A. Martin, C. L. Wilson, and M. Przybocki. An introduction to evaluating biometric systems. IEEE Computer,33(2):56-63, 2000.

Porter, On the " 30 error" criterion, ITT Industries

[23] Rosiek, T., \& Gupta, G. (2005). Generic Biometric System. West Virginia University Internal Technical Report. 
[24] Ross, A., \& Jain, A. (2004). Biometric Sensor Interoperability: A Case Study In Fingerprints. Paper presented at the meeting of the International ECCV Workshop on Biometric Authentication (BioAW). Prague, Czech Republic.

[25] Ross, A., Dass, S., \& Jain, A. (2005). A Deformable Model for Fingerprint Matching. Pattern Recognition, 38, 95-103.

[26] Schuckers, M. E., Hawley, A., \& Livingstone, K. (2004). A Comparison of Statistical Methods for Evaluating Matching Performance of a Biometric Identification Device- a Preliminary Report .

[27] Schuckers, M. E. (2003a). Estimation and sample size calculations for matching performance of biometric identification devices. Center for Identification Technology Research technical report.

. [28] Schuckers, M. E. "Using the beta-binomial distribution to assess performance of a biometric identification device,” International Journal of Image and Graphics (Special Issue on Biometrics) 3, pp. 523-529, July 2003.

[29] Speaks, D., Anderson, L., \& Hutson, J. (2004). Biometric Performanc Operational Testing and Reporting (M1/04-0216).

[30] Tabassi, E., Wilson, C., \& Watson, C. (2004). Fingerprint Image Quality (NIST 7151). Retrieved April 15, 2005 from http://fingerprint.nist.gov/NFIS/ir_7151.pdf

[31] Valencia, V. (2004). Biometric Testing: It's Not as Easy as You Think. http://WwW.biometrics.org/bc2004/CD/PDF_PROCEEDINGS/bc 076_ValenciaBief.pdf

[32] Wayman, J. "Technical testing and evaluation of biometric identification devices," in National Biometric Center Collected Works, J. Wayman, ed., pp. 67-89, 2000. 
[33] Wayman J. L.. Confidence interval and test size estimation for biometric data. In Proc. IEEE AutoID’99, pages 177-184, Oct. 1999.

[34] Wayman J. L., Error Rate Equations for the General Biometric System U.S. National Biometric Test Center

[35] Wayman, J.L. Technical testing and evaluation of biometric identification devices. Biometrics: Personal identification in networked society, edited by A.K. Jain, et al.,Kluwer, 2000, 345-368

[36] Wayman J. L., “A Scientific Approach to Evaluating Biometric Systems Using a Mathematical Methodology", Proc. CTST'97, pg. 477-492

[37] Wayman J. L., A. K. Jain, D. Maltoni, and D. Maio, Biometric Systems: Technology, Design and Performance Evaluation, Springer Verlag, 2005.

[38] Wilson, C., Hicklin, K., Bone, M., Korves, H., Grother, P., Ulery, B., et al. (2004). Fingerprint Vendor Technology Evaluation 2003: Summary of Results and Analysis Report (NISTIR 7123). 


\section{APPENDIX A SecuGen Hamster Optical Fingerprint Sensor}

\section{Specifications}

\begin{tabular}{|c|c|}
\hline Fingerprint Sensor & SecuGen FDU02 \\
\hline Dimensions (w/o stand) & $1.1^{\prime \prime}$ x $1.6 "$ x $2.9^{\prime \prime}(27$ x 40 x $73 \mathrm{~mm})$ \\
\hline Weight (w/o stand) & 3.5 oz. (100 g) \\
\hline Resolution & $500 \mathrm{dpi} \pm 0.2 \%$ \\
\hline Verification Time & Less than 1 second \\
\hline Operating Temperature & $32^{\circ}$ to $104^{\circ} \mathrm{F}\left(0^{\circ}\right.$ to $\left.40^{\circ} \mathrm{C}\right)$ \\
\hline Operating Humidity & $<90 \%$ relative, non-condensing \\
\hline Supply voltage & $5 \mathrm{~V} \pm 5 \%$ \\
\hline Interface & USB 1.1 \\
\hline Supported Operating Systems & $\begin{array}{l}\text { Windows } 2003 \text { / XP / } 2000 \text { / Me / } 98 \text { SE } \\
\text { - Download driver } \\
\text { Windows CE, CE .NET, Linux } \\
\text { - Available with SDK }\end{array}$ \\
\hline Certifications & FCC \\
\hline
\end{tabular}




\section{APPENDIX B Model: THWD-2 Digital Sling Psychrometer}

\section{Specifications}

- Range: Temp: $-4^{\circ} \mathrm{F}$ to $140^{\circ} \mathrm{F}\left(-20^{\circ} \mathrm{C}\right.$ to $\left.60^{\circ} \mathrm{C}\right) \&$ Humidity: $1 \% \mathrm{RH}$ to $99 \% \mathrm{RH}$

- Resolution: $0.1^{\circ} \mathrm{F}\left(0.1^{\circ} \mathrm{C}\right) \& 0.1 \% \mathrm{RH}$

- Accuracy: Temp: $\pm 1.5^{\circ} \mathrm{F}\left( \pm 0.8^{\circ} \mathrm{C}\right) \&$ Humidity $\pm 3 \% \mathrm{RH}$

- Data Memory Capacity: Manual Recording: 99 Data Sets (Direct reading from LCD display)

- Sampling Rate: 2 times/sec

- Operating Conditions: $32^{\circ} \mathrm{F}$ to $140^{\circ} \mathrm{F}\left(0^{\circ} \mathrm{C}\right.$ to $\left.60^{\circ} \mathrm{C}\right) \& 10$ to $80 \% \mathrm{RH}$

- Power Source: Three AAA (1.5V) Battery

- Battery Life: Approx. 200 hours

- $\quad$ Size: 6.7"(L) x 1.8"(W) x 0.8"(H) / 225mm(L) x 50mm(W) x 38mm(H)

- Weight: $0.22 \mathrm{Lb} /(100 \mathrm{~g})$

- Standard Accessories: Instruction Manual, Battery, Carrying Case 


\section{APPENDIX C Robotic Tripod}

\section{Detailed Hardware Specs}

- Maximum weight of webcam: $1.5 \mathrm{lbs}$

- Maximum angles of rotation: 160 degrees pan and 110 degrees tilt

- Maximum speed of movement: 100 degrees per second

- TrackerPod Interfaces to your PC via USB port

- TrackerPod powered by USB port or with optional external power supply if your computer's USB port cannot provide the needed current

\section{Minimum System Requirements}

- Operating system Windows ${ }^{\circledR}$ 98SE, ME, 2000, or XP.

- Processor $133 \mathrm{MHz}$ or faster; for best performance, $200 \mathrm{MHz}$ or faster recommended

- Memory 32MB RAM

- Interface available USB port

- Disk space 20MB available hard disk space 